\title{
Random analysis of coupled vehicle-bridge systems with local nonlinearity based on explicit time- domain method
}

Huan Huang ( $\sim$ happyhuang@scau.edu.cn )

South China Agricultural University https://orcid.org/0000-0002-0204-0888

Hengbin Zheng

South China Agricultural University

Jianliang Deng

South China Agricultural University

Wenxiong Li

South China Agricultural University

Yuyu Li

South China Agricultural University

\section{Research Article}

Keywords: Vehicle-bridge system, Hysteretic suspension, Equivalent linearization technique, Explicit timedomain method

Posted Date: April 12th, 2021

DOl: https://doi.org/10.21203/rs.3.rs-361197/v1

License: (1) This work is licensed under a Creative Commons Attribution 4.0 International License. Read Full License

Version of Record: A version of this preprint was published at Nonlinear Dynamics on January 18th, 2022. See the published version at https://doi.org/10.1007/s11071-021-07190-9. 


\title{
Random analysis of coupled vehicle-bridge systems with local nonlinearity based on explicit time-domain method
}

\author{
Huan Huang • Hengbin Zheng • Jianliang Deng • Wenxiong Li • Yuyu Li
}

\begin{abstract}
Based on the explicit time-domain method in conjunction with the equivalent linearization technique, an efficient analysis algorithm is developed for the random vibration analysis of the coupled vehicle-bridge system with local nonlinear components under the random irregular excitation from a bridge deck. With the coupled vehicle-bridge system divided into two subsystems, the equivalent linearized subsystem for the vehicle subsystem with the hysteretic suspension spring is constructed for the given time instant using the equivalent linearization technique. Then the dimension-reduction vibration analysis for the equivalent linearized coupled vehicle-bridge system can be carried out based on the time-domain explicit method, which has been proven to be highly efficient. The numerical example indicates that the proposed approach is of feasibility.
\end{abstract}

Keywords: Vehicle-bridge system; Hysteretic suspension; Equivalent linearization technique;

Explicit time-domain method

\section{Introduction}

In recent years, the random vibration problem of the coupled vehicle-bridge system has aroused the concern and interest of researchers[1-3]. Besides wind load and seismic load which cause random vibration of the coupled vehicle-bridge system, the random irregular excitation from a bridge deck or a rail is another significant excitation that can't be overlooked. As the coupled vehicle-bridge system is a time-variant system, even if the random irregular excitation is a stationary random process, the dynamic responses of the coupled vehicle-bridge system show typical nonstationary random processes. In addition, the nonlinear suspension spring with hysteretic properties is used in the suspension system of the vehicle subsystem for improving the ride comfort. Therefore, it is of great significance to investigate the random vibration of the coupled vehicle-bridge system that has local nonlinearity subjected to the random irregular excitation.

Thus far, different methods have been developed to evaluate the random responses of nonlinear systems, such as the Fokker-Planck-Kolmogorov equation method [3], the stochastic average method [5], the moment equation method [6], the equivalent linearization

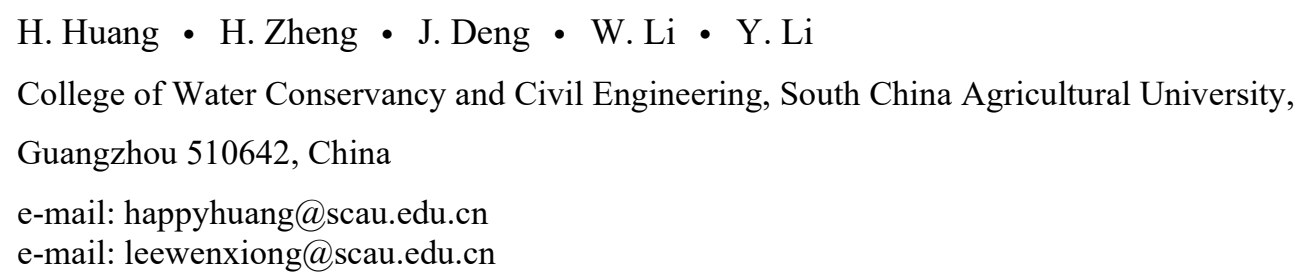


method [7], the Monte Carlo simulation method (MCS) [8], and so on. Most of them are limited to nonlinear systems with a limited number of degrees of freedom under stationary random excitations. The equivalent linearization method and the MCS are the only methods suitable for large-scale nonlinear systems. Although the MCS is a useful method for evaluating the random responses of the nonlinear systems, it is unpopular due to its high computational cost in interpretating the results. Even though, the MCS is often used to check the results of other methods. In consideration of the shortcoming of the methods mentioned above, the equivalent linearization method is widely accepted as an effective analytical tool for its high computational efficiency.

The basic idea of the equivalent linearization method is to substitute the original nonlinear system with a linear system according to a certain criterion. For the random vibration analysis of the coupled vehicle-bridge with nonlinear problem, Zhang and Knothe [9] extended the equivalent linearization method to the nonlinear contact geometry and nonlinear contact mechanics of the curved track under random track irregularities, and solved the linearized equation of motion by the power spectral density method (PSDM). Recently, Jin et al [10] studied the nonlinear wheel-rail contact of the coupled vehicle-bridge system under the rail irregularity using the equivalent linearization method, and obtained the statistical moments of the equivalent linear system based on the pseudo excitation method (PEM), which is a modified PSDM. The above research focused on the nonlinear wheel-rail contact geometry with the bridge subsystem remaining linear, which is in fact a local nonlinear problem. As the responses of the coupled vehicle-bridge system present nonstationary characteristic stated in paragraph one, it needs to transfer the random analysis of the equivalent linearized coupled vehicle-bridge system into a series of deterministic dynamic analysis at discretized frequency points [11] for the PSDM and the PEM. In another word, these methods are still relatively time-consuming when they are used to solve the equivalent linearized system with large-scale problems.

In order to improve the computational efficiency for calculating the nonstationary responses of the equivalent linearized system with large-scale problems, Su and his students [12-14] proposed an explicit time domain method (ETDM), which is devoted to solving the nonstationary responses of large-scale linear systems and equivalent linearized systems with high efficiency. The ETDM is a pure time-domain analysis method, in which the uncoupled treatment of physical and probabilistic evolution mechanism for the random vibration analysis of linear systems and equivalent linearized systems [15] are carried out, leading to the dimension-reduction analysis for random vibration problems. Therefore, it has higher computational efficiency than the PSDM and the PEM for which the two mechanisms are coupled together.

The purpose of this study is to extend the ETDM in conjunction with the equivalent 
linearization technique to the random vibration analysis of the coupled vehicle-bridge system. In this paper, the hysteretic nonlinear spring is used in the suspension system of the vehicle subsystem with the bridge subsystem remaining a linear system. The tight contact model between the wheel and rail is introduced in the coupled vehicle-bridge system. A numerical example indicates that the proposed method is feasible and effective.

\section{Equivalent linearization for the nonlinear coupled vehicle-bridge system}

\subsection{Restoring force model for hysteretic spring}

Based on the Bouc-Wen model, the restoring force of a hysteretic spring can be expressed as [16]

$$
\left\{\begin{array}{l}
f(y, z)=\alpha k y+(1-\alpha) k z \\
\dot{z}=A \dot{y}-\varphi|\dot{y}| z|z|^{\theta-1}-\psi \dot{y}|z|^{\theta}
\end{array}\right.
$$

where $y$ and $z$ are the real displacement and the hysteretic displacement, respectively; $\alpha$ denotes the ratio of post-yield to pre-yield stiffness and $k$ is the initial stiffness. As can be seen from Eq. (1), the hysteretic restoring force consists of two parts, the elastic force $\alpha k y$ and the hysteretic force $(1-\alpha) k z . \varphi, \psi, A$ and $\theta$ are the four parameters; $\varphi$ and $\psi$ are used to determine the hysteresis shape; $A$ represents the amplitude of the hysteretic force; $\theta$ refers to the smoothness from the elastic zone to plastic zone. By adjusting these parameters, one may obtain softening or hardening hysteretic restoring force models with different capacities of energy dissipation.

The equivalent linear equation for Eq. (1) can be rewritten as

$$
\left\{\begin{array}{l}
f(y, z)=\alpha k y+(1-\alpha) k z \\
\dot{z}=p \dot{y}+h z
\end{array}\right.
$$

where the two parameters $p$ and $h$ are equivalent coefficients determined by minimizing the mean square of the difference between Eqs. (1) and (2) at the specified instant. In this article, the value of $\theta$ is selected as 1 . When the system is subjected to Gaussian process, the response of the equivalent linear system will also follow Gaussian distributions. Under this condition, when $\theta=1$, the detailed expressions of $p$ and $h$ are expressed as [17]

$$
\left\{\begin{array}{l}
p=A-\sqrt{\frac{2}{\pi}}\left(\varphi \frac{\mathrm{E}(\dot{y} z)}{\sigma_{\dot{y}}}+\psi \sigma_{z}\right) \\
h=-\sqrt{\frac{2}{\pi}}\left(\varphi \sigma_{\dot{y}}+\psi \frac{\mathrm{E}(\dot{y} z)}{\sigma_{z}}\right)
\end{array}\right.
$$

Where $\mathrm{E}(\bullet)$ denotes the mathematical expectation.

In the following section, the hysteretic suspension spring, which is modelled by the Bouc-Wen model [16], is used in the simple vehicle subsystem of a coupled vehicle-bridge system. 


\subsection{Equivalent linear subsystem for the nonlinear vehicle subsystem}

Consider a simple mechanical vehicle-bridge model as shown in Fig. 1. The vehicle model is taken as a 2-degree-of-freedom (DOF) mass-spring system, in which the suspension spring is modelled by the Bouc-Wen model. The bridge model is taken as a simply supported uniform beam. $m_{1}$ and $m_{2}$ represent the mass of the vehicle body and the wheel, respectively. $k_{1}$ is the stiffness of the suspension spring modelled by the Bouc-Wen model, and $k_{2}$ is the stiffness of the wheel. $c$ is the damping of the suspension part. $y_{1}$ and $y_{2}$ represent the vertical displacements of the vehicle body and the wheel relative to the equilibrium position under the force of the gravity of the bridge subsystem, respectively. $y_{\mathrm{b}}$ is the displacement of the wheel-bridge contact point relative to the equilibrium position of the bridge subsystem. $v$ denotes the speed of the vehicle, and $x$ represents the distance of the vehicle travelling along the bridge.

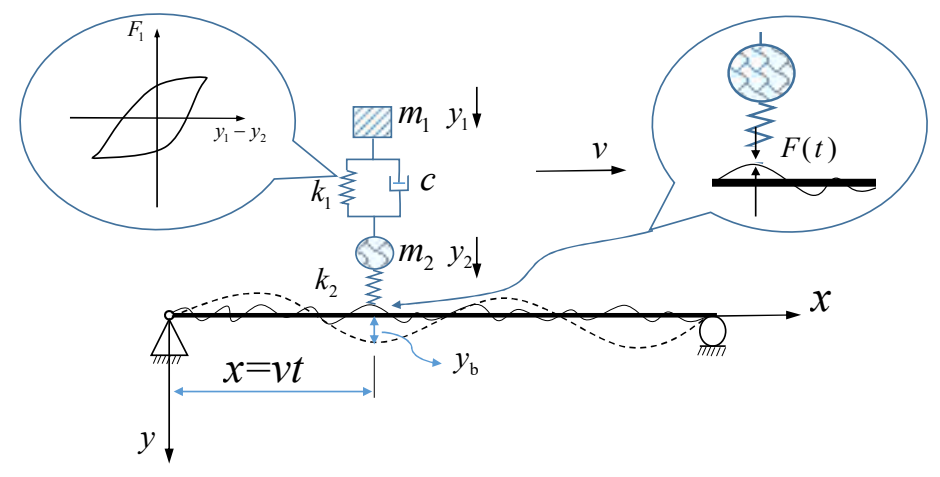

Fig. 1 Mechanical model of a coupled vehicle-bridge system

Introducing the vehicle-bridge contact force $F(t)$ as shown in Fig. 1, the equation of motion for the nonlinear vehicle subsystem at a specified time instant $\tau=t$ can be expressed as

$$
\begin{gathered}
\left\{\begin{array}{l}
m_{1} \ddot{y}_{1}+c\left(\dot{y}_{1}-\dot{y}_{2}\right)+\alpha k_{1}\left(y_{1}-y_{2}\right)+(1-\alpha) k_{1} z=0 \\
\dot{z}=A \dot{y}-\varphi|\dot{y}| z-\psi \dot{y}|z|
\end{array}\right. \\
m_{2} \ddot{y}_{2}-c\left(\dot{y}_{1}-\dot{y}_{2}\right)-\alpha k_{1}\left(y_{1}-y_{2}\right)-(1-\alpha) k_{1} z=-F(t)+\left(m_{1}+m_{2}\right) g
\end{gathered}
$$

where $g$ represents the gravitational acceleration. The vehicle-bridge contact force $F(t)$ is determined by the following compatibility equation

$$
y_{2}(t)-y_{\mathrm{b}}(t)-r(t)=-\frac{F(t)}{k_{2}}
$$

where $r(t)$ is the bridge undulation which represents the random rail irregularity, and it is 
supposed to be a homogeneous Guassian random process.

As stated in Section 2.1, Eq. (4) can be replaced by the following equivalent linear equation of motion

$$
\left\{\begin{array}{l}
m_{1} \ddot{y}_{1}+c \dot{y}_{1}-c \dot{y}_{2}+\alpha k_{1}\left(y_{1}-y_{2}\right)+(1-\alpha) k_{1} z=0 \\
\dot{z}=p(\tau) \dot{y}_{1-2}+h(\tau) z
\end{array}\right.
$$

where $\dot{y}_{1-2}=\dot{y}_{1}-\dot{y}_{2} . p$ and $h$ have the same form as Eq. (3), but they are only determined by the response for the specified time instant $\tau$, namely

$$
\left\{\begin{array}{l}
p(\tau)=A-\sqrt{\frac{2}{\pi}}\left(\varphi \frac{E\left[\dot{y}_{1-2}(\tau) z(\tau)\right]}{\sigma_{\dot{y}_{1-2}(\tau)}}+\psi \sigma_{z(\tau)}\right) \\
h(\tau)=-\sqrt{\frac{2}{\pi}}\left(\varphi \sigma_{\dot{y}_{1-2}(\tau)}+\psi \frac{E\left[\dot{y}_{1-2}(\tau) z(\tau)\right]}{\sigma_{z(\tau)}}\right)
\end{array}\right.
$$

Let $\boldsymbol{Y}_{\mathrm{v}}=\left[y_{1}, y_{2}, z\right]^{\mathrm{T}}$ with the superscript " $\mathrm{T}$ " for matrix transposition. Eq. (5) and Eq. (7) can be constructed into a matrix form which is expressed as follows

$$
\boldsymbol{M}_{\mathrm{v}} \ddot{\boldsymbol{Y}}_{\mathrm{v}}+\boldsymbol{C}_{\mathrm{v}}^{\mathrm{e}} \dot{\boldsymbol{Y}}_{\mathrm{v}}+\boldsymbol{K}_{\mathrm{v}}^{\mathrm{e}} \boldsymbol{Y}_{\mathrm{v}}=\boldsymbol{\Phi}\left[F(t)+\mathrm{G}_{\mathrm{v}}\right]
$$

where $\boldsymbol{M}_{\mathrm{v}}, \boldsymbol{C}_{\mathrm{v}}^{\mathrm{e}}$ and $\boldsymbol{K}_{\mathrm{v}}^{\mathrm{e}}$ are the extended mass matrix, the extended equivalent damping matrix and the extended equivalent stiffness matrix of the vehicle subsystem, and they are expressed as

$$
\boldsymbol{M}_{\mathrm{v}}=\left[\begin{array}{lll}
m_{2} & & \\
& m_{2} & \\
& & 0
\end{array}\right], \boldsymbol{C}_{\mathrm{v}}^{\mathrm{e}}=\left[\begin{array}{ccc}
c & -c & 0 \\
-c & c & 0 \\
-p & p & 1
\end{array}\right], \boldsymbol{K}_{\mathrm{v}}^{\mathrm{e}}=\left[\begin{array}{ccc}
\alpha k_{1} & -\alpha k_{1} & 0 \\
-\alpha k_{1} & \alpha k_{1} & 0 \\
0 & 0 & -h
\end{array}\right]
$$

Eq. (9) is the equation of motion for the equivalent linear system one finds at the specified time instant $\tau$. In Eq. (9), $\boldsymbol{\Phi}=[0,-1]^{\mathrm{T}}$ is the position vector an $\mathrm{G}_{\mathrm{v}}=\left(m_{1}+m_{2}\right) g$.

\subsection{Equation of motion for the bridge subsystem}

As can be seen from Fig. 1, a simply supported uniform beam is used in the bridge subsystem model. And the beam element is applied to the finite element model (FEM) of the bridge subsystem. The equation of motion for the linear bridge subsystem can be expressed in the following general form

$$
\boldsymbol{M}_{\mathrm{b}} \ddot{\boldsymbol{Y}}_{\mathrm{b}}+\boldsymbol{C}_{\mathrm{b}} \dot{\boldsymbol{Y}}_{\mathrm{b}}+\boldsymbol{K}_{\mathrm{b}} \boldsymbol{Y}_{\mathrm{b}}=\boldsymbol{L}_{\mathrm{b}}(x) F(t)
$$

where $\boldsymbol{M}_{\mathrm{b}}, \boldsymbol{C}_{\mathrm{b}}$ and $\boldsymbol{K}_{\mathrm{b}}$ are the global mass, damping and stiffness matrices of the bridge subsystem; $\boldsymbol{Y}_{\mathrm{b}}, \dot{\boldsymbol{Y}}_{\mathrm{b}}$ and $\ddot{\boldsymbol{Y}}_{\mathrm{b}}$ denote the absolute displacement, velocity and acceleration vectors relative to the equilibrium position caused by the gravity of the bridge subsystem; $F(t)$ is the wheel-bridge contact force; $\boldsymbol{L}_{\mathrm{b}}(x)$ is the position vector which is used to locate the position of $F(t)$ moving along the bridge subsystem.

Thus far, one has obtained the equations of motion for the equivalent linear vehicle 
subsystem and the bridge subsystem at the specified time instant $\tau$, respectively. The following section will discuss how to solve Eq. (9) and Eq. (11).

\section{Random analysis of the linearized coupled vehicle-bridge system by ETDM}

\subsection{Explicit expressions of dynamic responses for the equivalent linear vehicle}

\section{subsystem}

For the equation of motion of the equivalent linear vehicle subsystem corresponding to the concerned time instant $\tau$, as shown in Eq. (9), defines the following state vector as

$$
\boldsymbol{V}_{\mathrm{v}}=\left\{\begin{array}{c}
\boldsymbol{Y}_{\mathrm{v}} \\
\dot{\boldsymbol{Y}}_{\mathrm{v}}
\end{array}\right\}
$$

The recurrence formula of the state vector for the equivalent linear vehicle subsystem can be written as

$$
\boldsymbol{V}_{\mathrm{v}, i}=\boldsymbol{T}_{\mathrm{v}} \boldsymbol{V}_{\mathrm{v}, i-1}+\boldsymbol{Q}_{\mathrm{v} 1}\left[F_{i-1}+\mathrm{G}_{\mathrm{v}}\right]+\boldsymbol{Q}_{\mathrm{v} 2}\left[F_{i}+\mathrm{G}_{\mathrm{v}}\right] \quad(i=1,2, \cdots, n)
$$

where $n=\tau / \Delta t$ with $\Delta t$ being the time step; The subscripts " $i-1$ " and " $i$ " denote $t_{i-1}=(i-1) \Delta t$ and $t_{i}=i \Delta t$, respectively; $\boldsymbol{T}_{\mathrm{v}}, \boldsymbol{Q}_{\mathrm{v} 1}$ and $\boldsymbol{Q}_{\mathrm{v} 2}$ can be derived with the Newmark- $\beta$ integration scheme, which can be expressed as $[13,14]$

$$
\begin{aligned}
& \boldsymbol{T}_{\mathrm{v}}=\left[\begin{array}{ll}
\boldsymbol{H}_{11} & \boldsymbol{H}_{12} \\
\boldsymbol{H}_{21} & \boldsymbol{H}_{22}
\end{array}\right], \boldsymbol{Q}_{\mathrm{v} 1}=\left[\begin{array}{l}
\boldsymbol{R}_{1} \\
\boldsymbol{R}_{3}
\end{array}\right] \boldsymbol{\Phi}, \boldsymbol{Q}_{\mathrm{v} 2}=\left[\begin{array}{l}
\boldsymbol{R}_{2} \\
\boldsymbol{R}_{4}
\end{array}\right] \boldsymbol{\Phi} \\
& \boldsymbol{H}_{11}=\hat{\boldsymbol{K}}^{-1}\left(\boldsymbol{S}_{1}-\boldsymbol{S}_{3} \boldsymbol{M}_{\mathrm{v}}^{-1} \boldsymbol{K}_{\mathrm{v}}^{\mathrm{e}}\right), \boldsymbol{H}_{12}=\hat{\boldsymbol{K}}^{-1}\left(\boldsymbol{S}_{2}-\boldsymbol{S}_{3} \boldsymbol{M}_{\mathrm{v}}{ }^{-1} \boldsymbol{C}_{\mathrm{v}}^{\mathrm{e}}\right) \\
& \boldsymbol{H}_{21}=a_{3}\left(\boldsymbol{H}_{11}-\mathbf{I}\right)+a_{5} \boldsymbol{M}_{\mathrm{v}}^{-1} \boldsymbol{K}_{\mathrm{v}}^{\mathrm{e}}, \boldsymbol{H}_{22}=a_{3} \boldsymbol{H}_{12}-a_{4} \mathbf{I}+a_{5} \boldsymbol{M}_{\mathrm{v}}{ }^{-1} \boldsymbol{C}_{\mathrm{v}}^{\mathrm{e}} \\
& \boldsymbol{R}_{1}=\hat{\boldsymbol{K}}^{-1} \boldsymbol{S}_{3} \boldsymbol{M}_{\mathrm{v}}^{-1}, \boldsymbol{R}_{2}=\hat{\boldsymbol{K}}^{-1}, \boldsymbol{R}_{3}=a_{3} \boldsymbol{R}_{1}-a_{5} \boldsymbol{M}_{\mathrm{v}}^{-1}, \boldsymbol{R}_{4}=a_{3} \boldsymbol{R}_{2} \\
& \hat{\boldsymbol{K}}=\boldsymbol{K}_{\mathrm{v}}^{\mathrm{e}}+a_{0} \boldsymbol{M}_{\mathrm{v}}+a_{3} \boldsymbol{C}_{\mathrm{v}}^{\mathrm{e}} \\
& \boldsymbol{S}_{1}=a_{0} \boldsymbol{M}_{\mathrm{v}}+a_{3} \boldsymbol{C}_{\mathrm{v}}^{\mathrm{e}}, \boldsymbol{S}_{2}=a_{1} \boldsymbol{M}_{\mathrm{v}}+a_{4} \boldsymbol{C}_{\mathrm{v}}^{\mathrm{e}}, \boldsymbol{S}_{3}=a_{2} \boldsymbol{M}_{\mathrm{v}}+a_{5} \boldsymbol{C}_{\mathrm{v}}^{\mathrm{e}} \\
& a_{0}=1 /\left(\beta \Delta t^{2}\right), a_{1}=1 /(\beta \Delta t), a_{2}=1 /(2 \beta)-1 \\
& a_{3}=\gamma /(\beta \Delta t), a_{4}=\gamma / \beta-1, a_{5}=(\gamma / \beta-2) \Delta t / 2
\end{aligned}
$$

where $\mathbf{I}$ is the identity matrix; $\beta$ and $\gamma$ are two parameters used to control the Newmark- $\beta$ integration stability; In this study $\gamma=0.5$ and $\beta=0.25$ are used and the Newmark- $\beta$ method will be unconditionally stable.

Without loss of generality, $\mathbf{V}_{\mathrm{v}, 0}=\mathbf{V}_{\mathrm{v}}(0)=\mathbf{0}$ and $\ddot{y}_{1}(0)=\ddot{y}_{2}(0)=0$ are assumed. Then, the explicit expression for the state vector $\boldsymbol{V}_{\mathrm{v}}(\tau)$ at $t=\tau$ based on Eq. (11) can be written as

$$
\boldsymbol{V}_{\mathrm{v}}(\tau)=\boldsymbol{A}_{\mathrm{v}(n, 1)}\left(F_{1}+\mathrm{G}_{\mathrm{v}}\right)+\boldsymbol{A}_{\mathrm{v}(n, 2)}\left(F_{2}+\mathrm{G}_{\mathrm{v}}\right)+\cdots+\boldsymbol{A}_{\mathrm{v}(n, n)}\left(F_{n}+\mathrm{G}_{\mathrm{v}}\right)
$$

where $\boldsymbol{A}_{\mathrm{v}(n, j)}$ are the coefficient matrices and are expressed in closed forms as

$$
\boldsymbol{A}_{\mathrm{v}(n, \mathrm{n})}=\boldsymbol{Q}_{2}, \boldsymbol{A}_{\mathrm{v}(n, n-1)}=\boldsymbol{T} \boldsymbol{Q}_{2}+\boldsymbol{Q}_{1}, \boldsymbol{A}_{\mathrm{v}(n, n-j)}=\boldsymbol{T} \boldsymbol{A}_{\mathrm{v}(n, n-j+1)}(j=2,3, \cdots, n-1)
$$

Note that, from the physical point of view, the coefficient vectors $\boldsymbol{A}_{\mathrm{v}(n, n)}, \boldsymbol{A}_{\mathrm{v}(n, n-1)}, \cdots, \boldsymbol{A}_{\mathrm{v}(n, 2)}$ and $\boldsymbol{A}_{\mathrm{v}(n, 1)}$ exactly represent the state vectors at $t_{1}, t_{2}, \cdots, t_{n-1}$ and $t_{n}$, respectively, induced by an unit impulse of the wheel-bridge contact force $F(t)$ applied at $t=t_{1}$ [12]. Eq. 
(15) can be further expressed in a compact form as

$$
\boldsymbol{V}_{\mathrm{v}}(\tau)=\boldsymbol{A}_{\mathrm{v}[n]}\left(\boldsymbol{F}_{[n]}+\boldsymbol{G}_{[n]}\right)
$$

where $\boldsymbol{A}_{\mathrm{v}[n]}=\left[\begin{array}{llll}\boldsymbol{A}_{\mathrm{v}(n, 1)} & \boldsymbol{A}_{\mathrm{v}(n, 2)} & \cdots & \boldsymbol{A}_{\mathrm{v}(n, \mathrm{n})}\end{array}\right] ; \quad \boldsymbol{F}_{[n]}=\left[\begin{array}{lllll}F_{1} & F_{2} & \cdots & F_{n}\end{array}\right]^{\mathrm{T}}$ and $\boldsymbol{G}_{[n]}=\left[\begin{array}{llll}\mathrm{G}_{\mathrm{v}} & \mathrm{G}_{\mathrm{v}} & \cdots & \mathrm{G}_{\mathrm{v}}\end{array}\right]^{\mathrm{T}}$.

\subsection{Explicit expressions of dynamic responses for the bridge subsystem}

For the equation of motion of the bridge subsystem corresponding to the concerned time instant $\tau$, as shown in Eq. (11), defines the following state vector as

$$
\boldsymbol{V}_{\mathrm{b}}=\left\{\begin{array}{c}
\boldsymbol{Y}_{\mathrm{b}} \\
\dot{\boldsymbol{Y}}_{\mathrm{b}}
\end{array}\right\}
$$

Similar to the derivation of the dynamic responses for the equivalent linear vehicle subsystem above, the explicit expression for the state vector $\boldsymbol{V}_{\mathrm{b}}$ can also be written as

$$
\boldsymbol{V}_{\mathrm{b}}(\tau)=\boldsymbol{A}_{\mathrm{b}(n, 1)} F_{1}+\boldsymbol{A}_{\mathrm{b}(n, 2)} F_{2}+\cdots+\boldsymbol{A}_{\mathrm{b}(n, n)} F_{n}
$$

Note that, as the position of $F(t)$ travelled along the bridge subsystem changes with time, $A_{\mathrm{b}(n, 1)}$ represents the state vector at $t=t_{n}$ exerted by an unit impulse of the wheel-bridge contact force $F(t)$ applied at $t=t_{1} ; A_{\mathrm{b}(n, 2)}$ represents the state vector at $t=t_{n}$ exerted by an unit impulse of the wheel-bridge contact force $F(t)$ applied at $t=t_{2} ; \cdots ; A_{\mathrm{b}(n, \mathrm{n})}$ represents the state vector at $t=t_{n}$ exerted by an unit impulse of the wheel-bridge contact force $F(t)$ applied at $t=t_{\mathrm{n}}$. In other words, if there are $n$ time instants, there will be $n$ wheel-bridge contact points. Therefore, in order to obtain $\boldsymbol{A}_{\mathrm{b}(n, 1)}, A_{\mathrm{b}(n, 2)}, \cdots, A_{\mathrm{b}(n, \mathrm{n})}$, it needs $n$-time determinate dynamic history analyses for the bridge subsystem.

For simplicity, Eq. (19) can be also written as

$$
\boldsymbol{V}_{\mathrm{b}}(\tau)=\boldsymbol{A}_{\mathrm{b}[n]} \boldsymbol{F}_{[n]}
$$

where $\boldsymbol{A}_{\mathrm{b}[n]}=\left[\begin{array}{llll}\boldsymbol{A}_{\mathrm{b}(n, 1)} & \boldsymbol{A}_{\mathrm{b}(n, 2)} & \cdots & \boldsymbol{A}_{\mathrm{b}(n, \mathrm{n})}\end{array}\right]$ and $\boldsymbol{F}_{[n]}=\left[\begin{array}{llll}F_{1} & F_{2} & \cdots & F_{n}\end{array}\right]^{\mathrm{T}}$.

In fact, the wheel-bridge contact point will not always fall onto the node of the finite element. It may fall onto somewhere between the nodes of the same element. In such circumstances, the interpolation function for displacements of the beam elements and the equivalent node force needs to be taken into account for the derivation of $\boldsymbol{A}_{\mathrm{b}(n, j)}$ $(j=1,3, \cdots, n)$. For example, given that an unit impulse of the wheel-bridge contact force $F(t)$ at time $t=t_{k}(k \in[1,2, \cdots, n])$ fall onto somewhere between the nodes of a beam element, as is shown in Fig. 2, $d$ and $e$ are the nodes of the concerned beam element. Denote an unit impulse of the wheel-bridge contact force $F(t)$ at time $t=t_{k}(k \in[1,2, \cdots, n])$ as $\bar{F}\left(t_{k}\right)=1$. Based on the interpolation function for displacements of the beam elements, $\bar{F}\left(t_{k}\right)$ can be converted into the equivalent node forces, namely, $p_{d}$ 
and $r_{d}$ applied at node $d, p_{e}$ and $r_{e}$ applied at node $e$. Then, one can obtain $A_{\mathrm{b}(n, j)}$ through the state vector at $t=t_{k}$ exerted by the equivalent node forces $p_{d}, r_{d}, p_{e}$ and $r_{e}$.

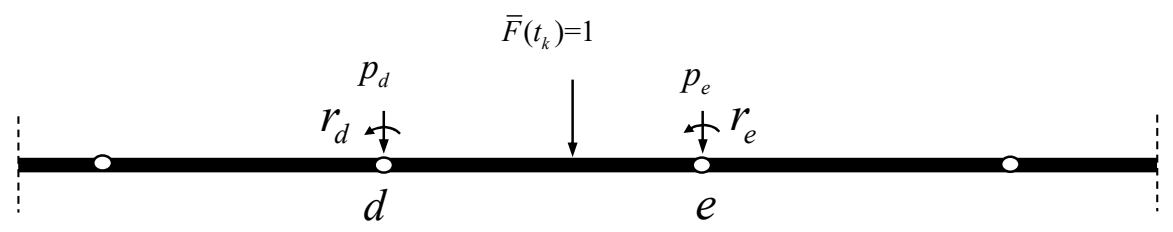

Fig. 2 Schematic diagram of the wheel-bridge contact position in FEM model

\subsection{Explicit expressions of contact forces}

It can be seen from Section 3.1 and Section 3.2, the responses of the vehicle subsystem and the bridge subsystem depend on the contact forces, which occur at the current time instant $\tau$ and all time instants before the current time instant $\tau$. Thus, the key point is to find these contact forces. In view of the following compatibility condition between the vehicle subsystem and the bridge subsystem as shown by Eq. (6), one can obtain the following expression, namely

$$
\boldsymbol{y}_{2[\mathrm{n}]}-\boldsymbol{y}_{\mathrm{b}[\mathrm{n}]}-\boldsymbol{r}_{[\mathrm{n}]}=-\frac{\boldsymbol{F}_{[\mathrm{n}]}}{k_{2}}
$$

where $\boldsymbol{r}_{[n]}=\left[\begin{array}{llll}r\left(x_{1}\right) & r\left(x_{2}\right) & \cdots & r\left(x_{n}\right)\end{array}\right]^{\mathrm{T}}$ with $x_{i}(1 \leq i \leq n)$ representing the distance of the vehicle travelling along the bridge; $y_{2[n]}$ is expressed as

$$
\boldsymbol{y}_{2[n]}=\boldsymbol{A}_{\mathrm{v} 2}\left(\boldsymbol{F}_{[n]}+\mathrm{G}_{[n]}\right)
$$

in which $\boldsymbol{y}_{2[n]}=\left[y_{2}\left(x_{1}, t_{1}\right) y_{2}\left(x_{2}, t_{2}\right) \cdots y_{2}\left(x_{n}, t_{n}\right)\right]^{\mathrm{T}} ; \boldsymbol{A}_{\mathrm{v} 2}$ is the matrix extracted from $\boldsymbol{A}_{\mathrm{v}[n]}$ accordingly; and $\boldsymbol{y}_{\mathrm{b}[\mathrm{n}]}$ can be written as

$$
\boldsymbol{y}_{\mathrm{b}[\mathrm{n}]}=A_{\mathrm{b}} \boldsymbol{F}_{[n]}
$$

in which $A_{\mathrm{b}}$ is the matrix extracted from $A_{\mathrm{b}[n]}$.

Substituting Eq. (22) and Eq. (23) into Eq. (21), one can obtain

$$
\boldsymbol{F}_{[n]}=\boldsymbol{A} r_{[n]}+\boldsymbol{B}
$$

where

$$
\left.\begin{array}{l}
\boldsymbol{A}=\left(\boldsymbol{A}_{\mathrm{v} 2}-\boldsymbol{A}_{\mathrm{b}}+\frac{\boldsymbol{I}}{k_{2}}\right)^{-1} \\
\boldsymbol{B}=-\boldsymbol{A} \boldsymbol{A}_{\mathrm{b}} \boldsymbol{G}_{[n]}
\end{array}\right\}
$$

Thus far, one has obtained the explicit expression of the contact forces $\boldsymbol{F}_{[n]}$ as shown by Eq. (24). In fact, Eq. (24) reflects the physical evolution mechanism of the equivalent linear 
vehicle-bridge coupled system. In other words, once the statistical moments of the contact forces $\boldsymbol{F}_{[n]}$ are obtained, it is easier to calculate the statistical moments of the responses for the equivalent linear coupled vehicle-bridge system.

\subsection{Random analysis}

The explicit expressions of dynamic responses for the equivalent linear vehicle subsystem and the linear bridge subsystem have been obtained in Sections 3.1-3.2. The statistical moments of dynamic responses will be discussed in detail in this section.

Based on Eq. (24), one can obtain the mean vector of the contact forces $\boldsymbol{F}_{[n]}$, respectively, namely

$$
E\left(\boldsymbol{F}_{[n]}\right)=\boldsymbol{A} E\left(\boldsymbol{r}_{[n]}\right)+\boldsymbol{B}
$$

where $E\left(\boldsymbol{F}_{[n]}\right)$ is the mean vector of the contact forces $\boldsymbol{F}_{[n]} ; E\left(\boldsymbol{r}_{[n]}\right)$ denotes the mean vectors of the bridge undulation, which can be expressed as follows

$$
E\left(\boldsymbol{r}_{[n]}\right)=\left[\begin{array}{llll}
\mu_{r}\left(x_{1}\right) & \mu_{r}\left(x_{2}\right) & \cdots & \mu_{r}\left(x_{n}\right)
\end{array}\right]^{\mathrm{T}}
$$

where $\mu_{r}\left(x_{i}\right)=E\left[r\left(x_{i}\right)\right](1 \leq i \leq n)$ is the mean function of the bridge undulation.

Similarly, the correlation matrix of the contact forces $\boldsymbol{F}_{[n]}$ can be given as follows

$$
\operatorname{cov}\left(\boldsymbol{F}_{[n]}, \boldsymbol{F}_{[n]}\right)=\boldsymbol{A} \operatorname{cov}\left(\boldsymbol{r}_{[n]}, \boldsymbol{r}_{[n]}\right) \boldsymbol{A}^{\mathrm{T}}
$$

where $\operatorname{cov}\left(\boldsymbol{r}_{[n]}, \boldsymbol{r}_{[n]}\right)$ is the correlation matrix of the bridge undulation expressed by

$$
\operatorname{cov}\left(\boldsymbol{r}_{[n]}, \boldsymbol{r}_{[n]}\right)=\left[\begin{array}{ccc}
R_{r}\left(x_{1}, x_{1}\right)-\mu_{r}^{2}\left(x_{1}\right) & R_{r}\left(x_{1}, x_{2}\right)-\mu_{r}\left(x_{1}\right) \mu_{r}\left(x_{2}\right) & \cdots \\
R_{r}\left(x_{2}, x_{1}\right)-\mu_{r}\left(x_{2}\right) \mu_{r}\left(x_{1}\right) & R_{r}\left(x_{2}, x_{2}\right)-\mu_{r}^{2}\left(x_{2}\right) & \cdots \\
\vdots & \vdots & \ddots \\
R_{r}\left(x_{n}, x_{1}\right)-\mu_{r}\left(x_{n}\right) \mu_{r}\left(x_{1}\right) & R_{r}\left(x_{n}, x_{2}\right)-\mu_{r}\left(x_{n}\right) \mu_{r}\left(x_{2}\right) & \cdots \\
& R_{r}\left(x_{1}, x_{n}\right)-\mu_{r}\left(x_{1}\right) \mu_{r}\left(x_{n}\right) \\
R_{r}\left(x_{2}, x_{n}\right)-\mu_{r}\left(x_{2}\right) \mu_{r}\left(x_{n}\right) \\
\vdots \\
R_{r}\left(x_{n}, x_{n}\right)-\mu_{r}^{2}\left(x_{n}\right)
\end{array}\right]
$$

where $R_{r}\left(x_{i}, x_{j}\right)(1 \leq i \leq n, 1 \leq j \leq n)$ is the correlation function of the bridge undulation.

For the case when local nonlinear behaviors exists in the vehicle subsystem, only a small number of statistical moments, e.g., $E\left[\dot{y}_{1-2}(\tau) z(\tau)\right] 、 \sigma_{\dot{y}_{1-2}(\tau)}$ and $\sigma_{z(\tau)}$, need to be calculated in Eq. (8) in order to obtain the equivalent linear vehicle-bridge coupled system. Based on Eq. (17), one can pick out $\dot{y}_{1}(\tau), \dot{y}_{2}(\tau)$ and $z(\tau)$ from Eq. (17), namely

$$
\begin{gathered}
\dot{y}_{1}(\tau)=\boldsymbol{A}_{\dot{y}_{1}[n]}\left(\boldsymbol{F}_{[n]}+\boldsymbol{G}_{[n]}\right) \\
\dot{y}_{2}(\tau)=\boldsymbol{A}_{\dot{y}_{2}[n]}\left(\boldsymbol{F}_{[n]}+\boldsymbol{G}_{[n]}\right) \\
z(\tau)=\boldsymbol{A}_{z[n]}\left(\boldsymbol{F}_{[n]}+\boldsymbol{G}_{[n]}\right)
\end{gathered}
$$

From Eq. (30) and Eq. (31), one can easily obtain the relative velocity $\dot{y}_{1-2}$ as follows 


$$
\dot{y}_{1-2}(\tau)=\boldsymbol{A}_{\dot{y}_{1-2}[n]}\left(\boldsymbol{F}_{[n]}+\boldsymbol{G}_{[n]}\right)
$$

where $\boldsymbol{A}_{\dot{y}_{1-2}[n]}=\boldsymbol{A}_{\dot{y}_{1}[n]}-\boldsymbol{A}_{\dot{y}_{2}[n]}$.

Based on Eq. (32) and Eq. (33), one can obtain the mean of $\dot{y}_{1-2}$ and $z(\tau)$, respectively

$$
\begin{aligned}
\mu_{\dot{y}_{1-2}}(\tau) & =\boldsymbol{A}_{\dot{y}_{1-2}[n]} E\left[\boldsymbol{F}_{[n]}\right] \\
\mu_{z(\tau)}(\tau) & =\boldsymbol{A}_{z(\tau)} E\left[\boldsymbol{F}_{[n]}\right]
\end{aligned}
$$

Then, $E\left[\dot{y}_{1-2}(\tau) z(\tau)\right] 、 \quad \sigma_{\dot{y}_{1-2}(\tau)}$ and $\sigma_{z(\tau)}$ can be written as

$$
\begin{gathered}
E\left[\dot{y}_{1-2}(\tau) z(\tau)\right]=\boldsymbol{A}_{\dot{y}_{1-2}[n]} \operatorname{cov}\left(\boldsymbol{F}_{[n]}, \boldsymbol{F}_{[n]}\right) \boldsymbol{A}_{z[n]}^{\mathrm{T}} \\
\sigma_{\dot{y}_{1-2}(\tau)}=\sqrt{\boldsymbol{A}_{\dot{y}_{1-2}[n]} \operatorname{cov}\left(\boldsymbol{F}_{[n]}, \boldsymbol{F}_{[n]}\right) \boldsymbol{A}_{\dot{y}_{1-2}[n]}^{\mathrm{T}}-\mu_{\dot{y}_{1-2}}^{2}(\tau)} \\
\sigma_{z(\tau)}=\sqrt{\boldsymbol{A}_{z[n]} \operatorname{cov}\left(\boldsymbol{F}_{[n]}, \boldsymbol{F}_{[n]}\right) \boldsymbol{A}_{z[n]}^{\mathrm{T}}-\mu_{z(\tau)}^{2}(\tau)}
\end{gathered}
$$

Once $E\left[\dot{y}_{1-2}(\tau) z(\tau)\right] 、 \sigma_{\dot{y}_{1-2}(\tau)}$ and $\sigma_{z(\tau)}$ are obtained, the equivalent coefficients $p(\tau)$ and $h(\tau)$ can be updated through Eq. (8), and the equivalent linear equation of motion shown in Eq. (9) can also be updated accordingly. Repeat this calculation until the statistical moments in $E\left[\dot{y}_{1-2}(\tau) z(\tau)\right] 、 \quad \sigma_{\dot{y}_{1-2}(\tau)}$ and $\sigma_{z(\tau)}$ are convergent. Then, one can calculate the statistical moments of the other required responses of the bridge subsystem. Since one has obtained the explicit expression of dynamic responses of the bridge subsystem shown by Eq. (20), any dynamic response which one concerns can be extracted from Eq. (20). For instance, one may concern a dynamic response of the bridge, the required response can be given as follows

$$
v_{\mathrm{b}}(\tau)=\boldsymbol{a}_{\mathrm{b}[n]} \boldsymbol{F}_{[n]}
$$

where $v_{\mathrm{b}}(\tau)$ is the concerned response extracted from $\boldsymbol{V}_{\mathrm{b}}(\tau)$ and $\boldsymbol{a}_{\mathrm{b}[n]}$ is the corresponding row vector extracted from $\boldsymbol{A}_{\mathrm{b}[n]}$. Then one can obtain the 1-st and the 2-nd order statistical moments of $v_{\mathrm{b}}(\tau)$ as

$$
\begin{gathered}
\mu_{v_{\mathrm{b}}}(\tau)=E\left[v_{\mathrm{b}}(\tau)\right]=\boldsymbol{a}_{\mathrm{b}[n]} E\left[\boldsymbol{F}_{[n]}\right] \\
E\left[v_{\mathrm{b}}^{2}(\tau)\right]=\boldsymbol{a}_{\mathrm{b}[n]} \operatorname{cov}\left(\boldsymbol{F}_{[n]}, \boldsymbol{F}_{[n]}\right) \boldsymbol{a}_{\mathrm{b}[n]}^{\mathrm{T}}
\end{gathered}
$$

The variance of $v_{\mathrm{b}}(\tau)$ can expressed as

$$
\sigma_{v_{\mathrm{b}}}^{2}(\tau)=E\left[v_{\mathrm{b}}^{2}(\tau)\right]-E^{2}\left[v_{\mathrm{b}}(\tau)\right]
$$

Thus far, one has obtained the required responses based on Eqs. (40)-(42). Then one can move on to the next time instant until the responses at all time instants concerned are obtained.

\section{Solution procedure for the equivalent linear coupled vehicle-bridge system}

To illustrate the above proposed method for the nonlinear coupled vehicle-bridge system 
under the bridge undulation, an iterative solution procedure is given as follows:

(1) Calculate the explicit expression of the dynamic responses for the linear bridge subsystem shown by Eq. (20) based on Eq. (11) at all time instants.

(2) Assign an initial values of $E\left[\dot{y}_{1-2}(\tau) z(\tau)\right], \sigma_{\dot{y}_{1-2}(\tau)}$ and $\sigma_{z(\tau)}$ to equivalent coefficients $p(\tau)$ and $h(\tau)$ (the converged results for the previous time instance may be used).

(3) Solve the equivalent linearized vehicle subsystem with Eqs. (9) and (13), and obtain the explicit expression of dynamic responses for the equivalent linearized vehicle subsystem shown by Eq. (17).

(4) Calculate the explicit expression of the contact forces expressed by Eq. (24) based on Eq. (25).

(5) Obtain the mean vector and the correlation matrix of the contact forces $\boldsymbol{F}_{[n]}$ based on Eq. (26) and Eq. (28).

(6) Calculate $E\left[\dot{y}_{1-2}(\tau) z(\tau)\right] 、 \quad \sigma_{\dot{y}_{1-2}(\tau)}$ and $\sigma_{z(\tau)}$ based on Eqs. (36)-(38).

(7) Substitute the new values of $E\left[\dot{y}_{1-2}(\tau) z(\tau)\right], \sigma_{\dot{y}_{1-2}(\tau)}$ and $\sigma_{z(\tau)}$ into $p(\tau)$ and $h(\tau)$ at time instant $\tau$, and update the equivalent coefficients.

(8) Repeat steps (2)-(7) until the statistical moments are convergent.

(9) Calculate the statistical moments of any concerned response based on Eqs. (40)-(42).

(10) Move on to the next time instant. Repeat (2)-(9) until the required statistical moments of responses at all the time instants concerned are obtained.

For clarity, the above solution procedure based on the proposed method can be vividly depicted by the following flowchart as shown in Fig. 2.

\section{Numerical examples}

In this following numerical example, the parameters of the coupled vehicle-bridge system model are selected from Refs. [1] and [18]. Data for the vehicle-bridge model in Fig. 1 is given as follows: $E I=2658069 \mathrm{kN} \cdot \mathrm{m}^{2}, \rho A=6067 \mathrm{kN} \cdot \mathrm{m}^{2}, \quad m_{1}=12000 \mathrm{~kg}, \quad m_{2}=500 \mathrm{~kg}$, $k_{1}=280000 \mathrm{~N} / \mathrm{m} ; k_{2}=156000 \mathrm{~N} / \mathrm{m}$. The stiffness reduction ratio for the suspension spring $k_{1}$ is set to be $\alpha=0.5$. The parameters of the hysteretic displacement are $A_{i}=1, \varphi_{i}=400 \mathrm{~m}^{-1}$, $\psi_{i}=300 \mathrm{~m}^{-1}$ and $\theta_{i}=1$. The bridge length is set to be $l=40 \mathrm{~m}$. The vehicle is travelling at speed $v=20 \mathrm{~m} / \mathrm{s}$. The bridge undulation $r(t)$ is supposed to be a homogeneous Guassian random process with a zero mean value and its power spectral density is expressed by

$$
S(\omega)=\frac{1}{\pi} \frac{4 \gamma \beta \chi \omega_{0}^{2}}{\left(\omega_{0}^{2}-\omega^{2}\right)^{2}+4 \beta^{2} \omega^{2}}
$$

where $\omega_{0}^{2}=\beta^{2}+\chi^{2}$, and $\beta=0.1, \quad \chi=0.3, \gamma=1 \mathrm{~cm}^{2}$. Since $S(\omega)$ decreases rapidly when 
$\omega \geq \omega_{0}$, the frequency range of integration is set to be $\omega \in(-10,10) \operatorname{rad}$ which is good enough for this example.

In this example, the proposed method and the Monte Carlo simulation (MCS) are used for the random vibration analysis of the nonlinear coupled vehicle-bridge system subjected to random rail irregularities. The bridge subsystem is divided into 20 plane beam elements,

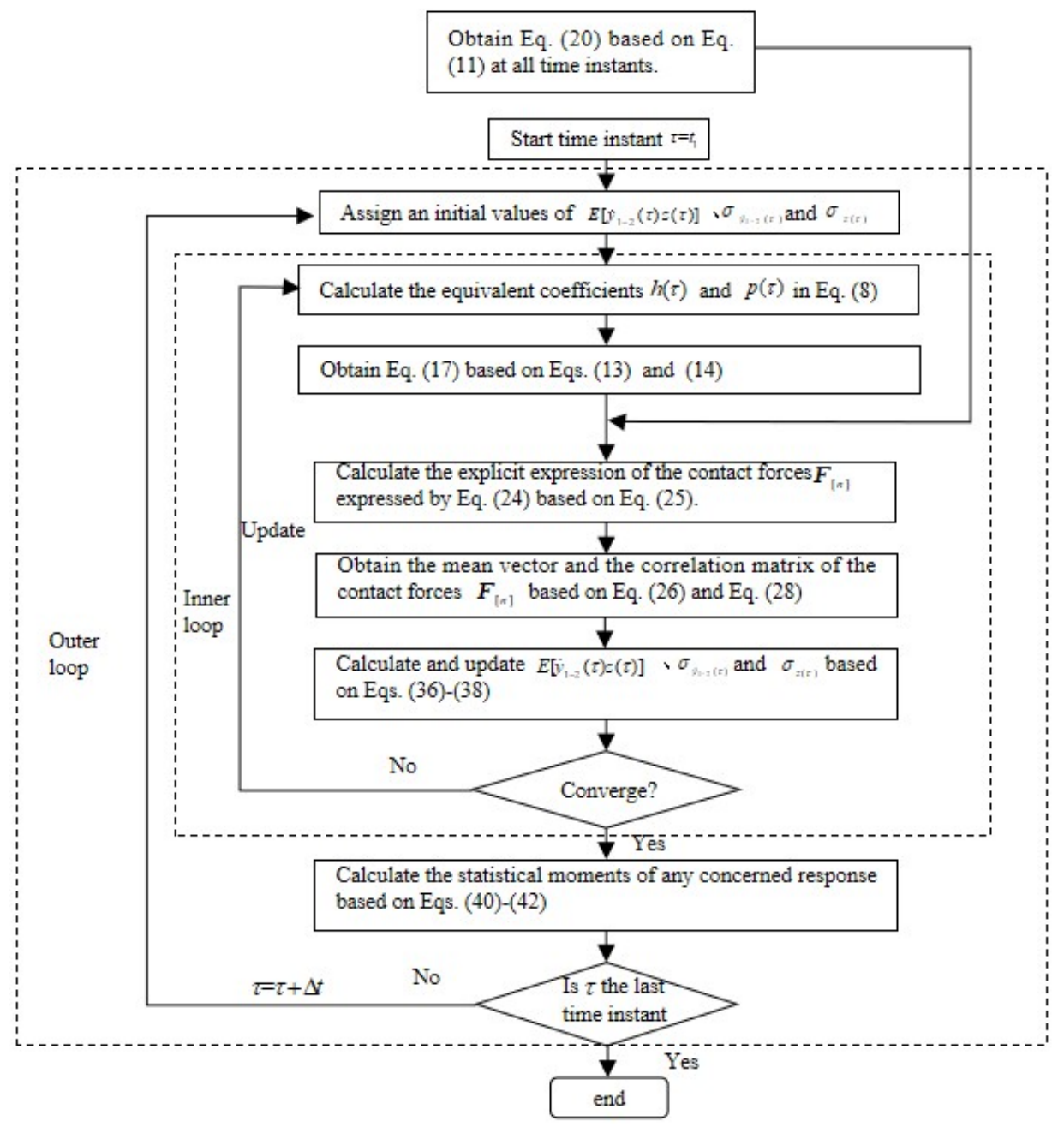

Fig. 2 The flowchart of the solution procedure based on the proposed method

which are enough for this model. The duration of the vehicle crossing the bridge is $T=(40 / 20) \mathrm{s}=2 \mathrm{~s}$. The time step used for the proposed method is set to be $\Delta t=0.02 \mathrm{~s}$. The number of samples is $N=1,000$ for MCS, and the time step is set to be $\Delta t=0.002 \mathrm{~s}$ for the nonlinear vehicle subsystem and $\Delta t=0.02 \mathrm{~s}$ for the linear bridge subsystem, respectively.

The mean time history and the standard deviation of the mid-span displacement of the bridge subsystem are shown in Figs. 3 and 4, respectively, from which it can be seen that the results of the proposed method and the MCS are in good agreement. In addition, the mean time history and the standard deviation time history of the mid-span displacement for linear 
coupled vehicle-bridge system from Ref. [18] are given in this example as shown in Figs 3 and 4. As can be seen from Figs 3 and 4, there is a little difference for the mean time history between the nonlinear system and the linear system. But the difference for the standard deviation between the nonlinear system and the linear system can't be neglected. It shows the hysteretic suspension spring influences the responses of the coupled vehicle-bridge system.

As the acceleration of the vehicle body plays an important role in the ride comfort. Figs 5 and 6 give the mean value time history and the standard time history of the vertical acceleration $a$ for the vehicle body $m_{1}$. The results from Ref. 18 are also demonstrated in figures 5 and 6 . It shows that the vertical acceleration of the vehicle body decreases when the suspension system exhibits hysteretic properties.

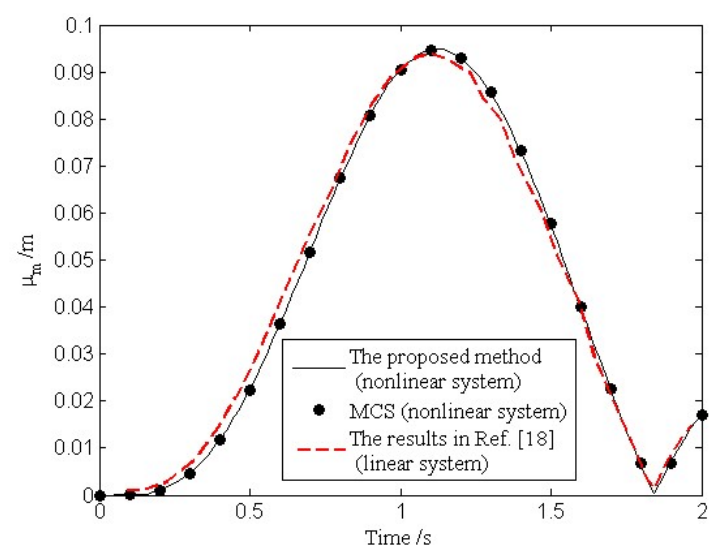

Fig. 3 The mean time history for the mid-span displacement $(\alpha=0.5)$

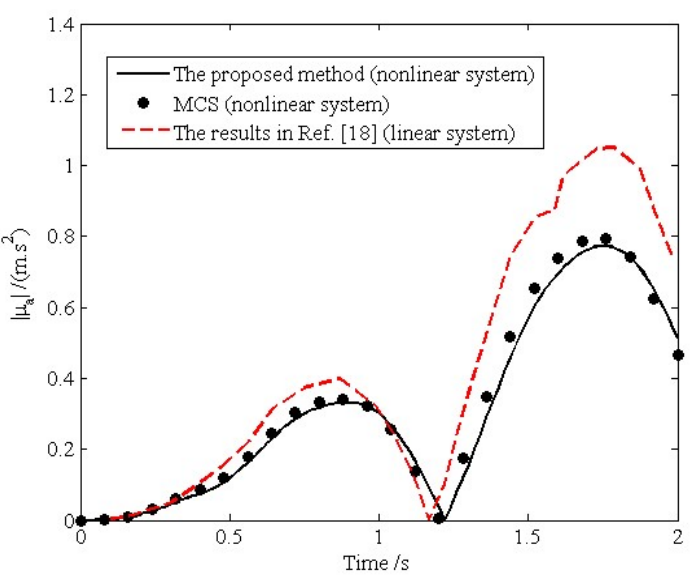

Fig. 5 The mean value time history of vertical acceleration $a$ of vehicle body $m_{1}(\alpha=0.5)$

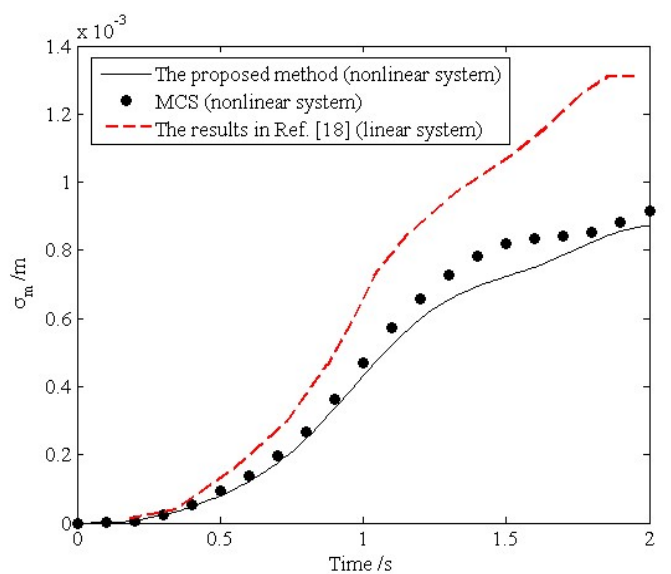

Fig. 4 The standard time history for the mid-span displacement $(\alpha=0.5)$

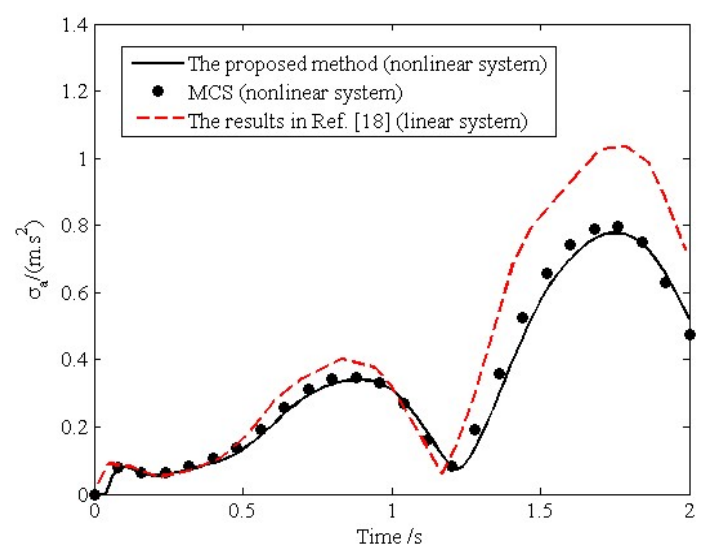

Fig. 6 The standard time history of vertical acceleration $a$ of vehicle body $m_{1} \quad(\alpha=0.5)$ 
Furthermore, three cases for the velocity, that is $v=16 \mathrm{~m} / \mathrm{s}, 20 \mathrm{~m} / \mathrm{s}$ and $40 \mathrm{~m} / \mathrm{s}$, are taken into account to investigate the influence of the velocity of the vehicle traveling, respectively. The mean time histories and the standard deviation histories of the mid-span displacement of the bridge subsystem are shown in Figs. 7 and 8 with different velocities. The same are the mean value time histories and the standard time histories of the vertical acceleration $a$, which are presented in Figs. 9 and 10. It can be seen from Figs. 7-10 that good agreement is also achieved for the statistical moments obtained by the proposed method and the MCS.

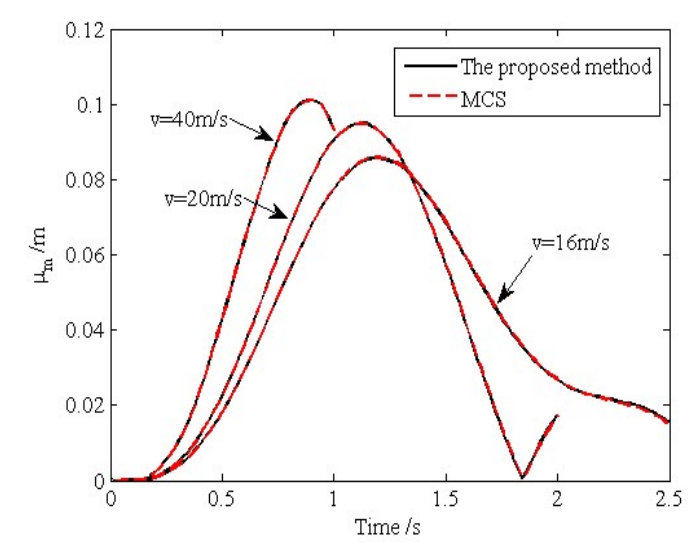

Fig. 7 The mean time histories for the mid-span displacement with different velocities $(\alpha=0.5)$

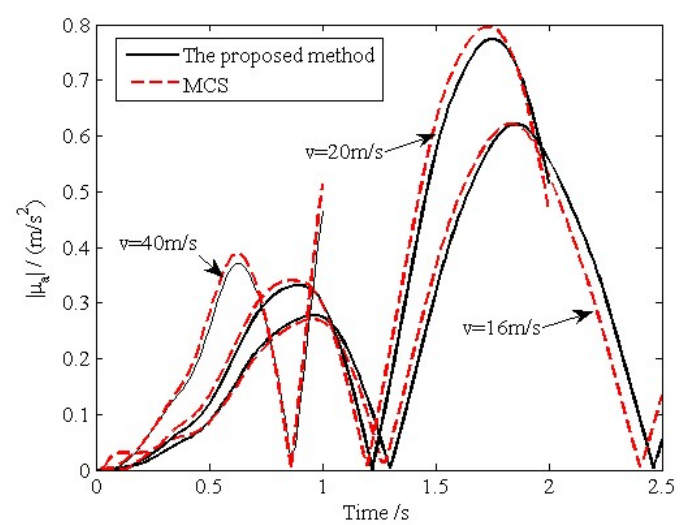

Fig. 9 The mean value time histories of vertical acceleration $a$ of vehicle body $m_{1}$ with different velocities $(\alpha=0.5)$

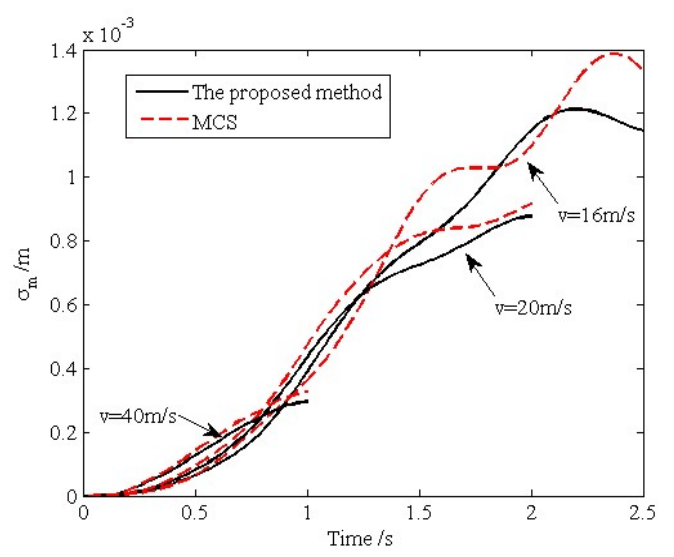

Fig. 8 The standard time histories for the mid-span displacement with different velocities $(\alpha=0.5)$

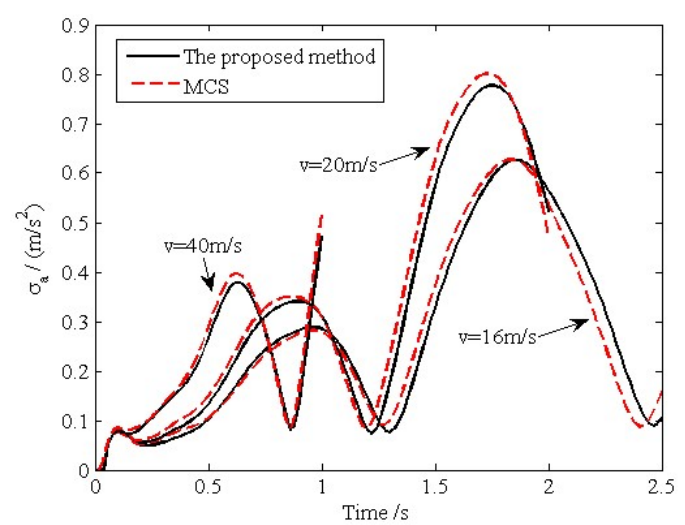

Fig. 10 The standard time histories of vertical acceleration $a$ of vehicle body $m_{1}$ with different velocities $(\alpha=0.5)$

Lastly, in order to investigate the influence of the hysteretic suspension spring on the responses of the coupled vehicle-bridge system, the stiffness reduction ratio for the suspension spring $k_{1}$ is set to be $\alpha=0.8,0.5$ and 0.2 , respectively. The time histories of the concerned statistical moments for the bridge subsystem and the vehicle subsystem are shown in Figs. 11-14, respectively. Obviously, Figs. 11-14 show that the relative error of the 
proposed method increases apparently with the increase in nonlinear levels of the hysteretic suspension spring. This mainly attributes to the Guassian assumption of responses adopted in the equivalent linearization technique. When the stiffness reduction is severe, e.g., $\alpha=0.2$, the responses may depart severely from the Guassian distribution, resulting in bad calculating accuracy.

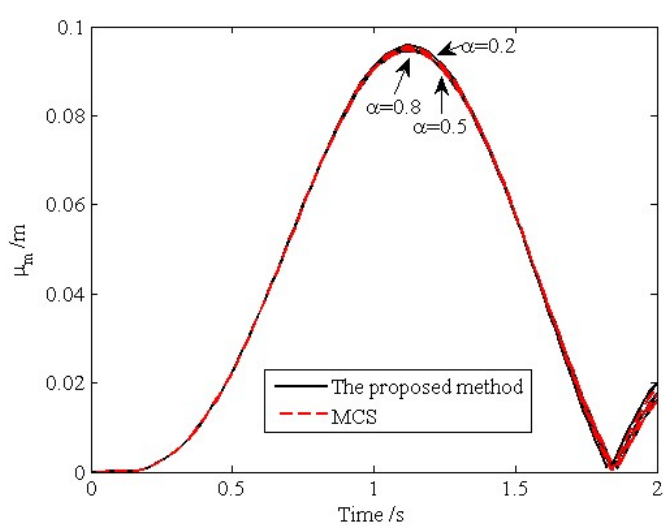

Fig. 11 The mean time histories for the mid-span displacement with $\alpha=0.8,0.5$ and 0.2

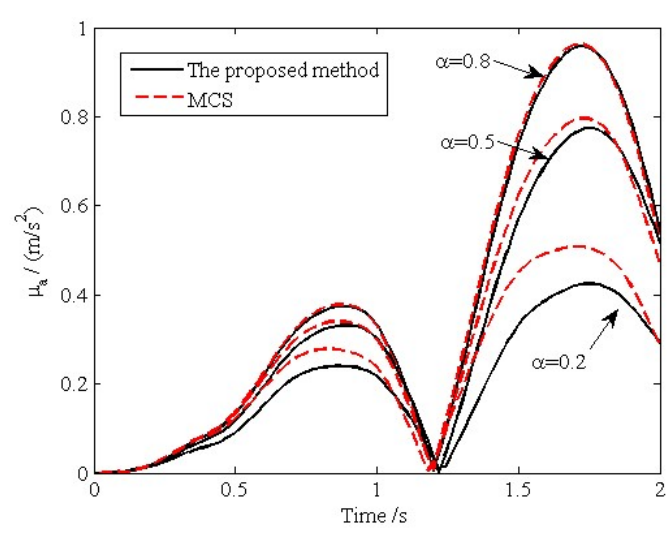

Fig. 13 The mean value time histories of vertical acceleration $a$ of vehicle body $m_{1}$ with $\alpha=0.8,0.5$ and 0.2

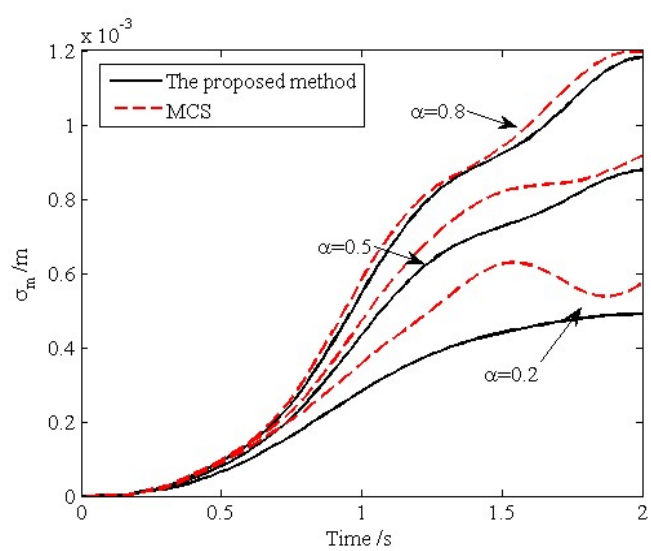

Fig. 12 The standard time histories for the mid-span displacement with $\quad \alpha=0.8,0.5$ and 0.2

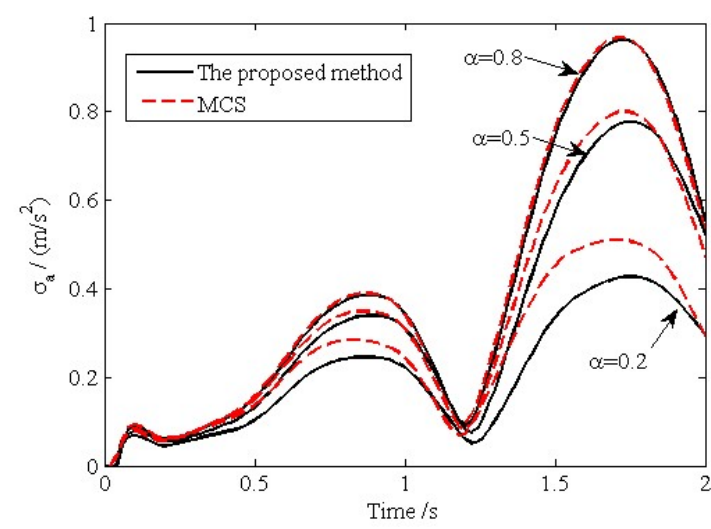

Fig. 14 The standard time histories of vertical acceleration $a$ of vehicle body $m_{1}$ with $\alpha=0.8, \quad 0.5$ and 0.2

\section{Conclusions}

An efficient method for the random vibration analysis of the coupled vehicle-bridge system with the local nonlinear problem is developed by implementing the ETDM in conjunction with the equivalent linearization technique. Using the equivalent linearization technique, the hysteretic vehicle subsystem is replaced by an equivalent linear vehicle subsystem. The dimension-reduction analysis for the responses involved with the local nonlinear components 
and the responses concerned can be carried out efficiently based on the ETDM. Numerical results show that the hysteretic suspension system can reduce the dynamic responses of the coupled vehicle-bridge system, and improve the ride comfort through decreasing the acceleration of the vehicle body. The proposed method will be extended to more scenarios of the coupled vehicle-bridge system in the future, such as sensitivity analysis, design optimization and so on.

\section{Acknowledgements}

The project is funded by the Natural Science Foundation of Guangdong Province, China (Grant No. 2020A1515010611).

\section{Compliance with ethical standards}

Conflicts of interest The authors declare that they have no conflict of interest.

\section{References}

1. Li J.-Q, Leng X.-L, Fang T . Evolutionary random response problem of a coupled vehicle-bridge system. Arch. Appl. Mech. 72(6/7), 536-544 (2002)

2. Pablo A, Zhang N, José M.-G, He X, Miguel Á.-A, Javier O. Consideration of nonlinear wheel-rail contact forces for dynamic vehicle-bridge interaction in high-speed railways. J. Sound Vib. 332(5), 1231-1251 (2013)

3. Zhang Z, Zhang Y, Lin J.-h, Zhao Y, Howson W.-P, Williams F.-W. Random vibration of a train traversing a bridge subjected to traveling seismic waves. Eng. Struct. 33(12), 3546-3558 (2011)

4. Zhu W.-Q. Nonlinear stochastic dynamic and control in Hamiltonian formulation. Appl. Mech. Rev. 59(4), 230-245 (2006)

5. Zeng Y, Zhu W.-Q. Stochastic averaging of quasi-linear systems driven by Poisson white noise. Probab. Eng. Mech. 25(1): 99-107 (2010)

6. Crandall S.-H. Non-Gaussian closure for random vibration of nonlinear oscillator. Int. J. Non. Mech. 15(4-5), 303-313 (1980)

7. Crandall S.-H. A half-century of stochastic equivalent linearization. Struct. Control Health Monit. $13,27-40$ (2006)

8. Proppe C, Pradlwarter H.-J , Schuëller G.-I . Equivalent linearization and Monte Carlo simulation in stochastic dynamics. Probab. Eng. Mech. 18(1), 1-15 (2003)

9. Zhang J.-J, Klaus K. Statistical linearization of wheel/rail contact nonlinearities for investigation of curving behaviour with random track irregularities. Vehicle Sys. Dyn. Sup. 25, 731-745 (1996)

10. Jin Z.-B, Li X.-Z, Zhu Y, Qiang S.-Z. Random vibration analysis of nonlinear vehicle-bridge dynamic interactions. J China Railway Soc. 39(9), 109-116 (2017) (in Chinese)

11. Lu F, Lin J.-H , Kennedy D, Williams F.-W. An algorithm to study non-stationary random vibrations of vehicle-bridge systems. Comp. Struct., 87(3-4), 177-185 (2009)

12. $\mathrm{Su} \mathrm{C}, \mathrm{Xu} \mathrm{R}$. Random vibration analysis of structures by a time-domain explicit formulation 
method. Struct. Eng. Mech. 52(2), 239-260 (2014)

13. Su C, Huang H, Ma H.-T. Fast Equivalent Linearization Method for Nonlinear Structures under Nonstationary Random Excitations. J. Eng. Mech. 142(8), 04016049 (2016)

14. Su C, Xian J.-H, Huang H. An iterative equivalent linearization approach for stochastic sensitivity analysis of hysteretic systems under seismic excitations based on explicit time-domain method. Comp. Struct. 242(1), 160396 (2020)

15. Su C, Sang X.-L, Huang H, On physical Mechanism and probabilistic evolution in random vibration of structures. Symposium on reliability of engineering system, Hangzhou (2015)

16. Wen Y.-K. Method for Random Vibration of Hysteretic Systems. J. Eng. Mech. Div. 102(2), 249-263 (1976)

17. Roberts J.-B, Spanos P.-D. Random Vibration and Statistical Linearization. Dover Publication, New York (2003)

18. Su C, Zhong C.-Y, Zhou L.-C. Random vibration analysis of coupled vehicle-bridge system with the explicit time-domain method. Appl. Math. Mech., 38(1), 99-108 (2017) (in Chinese) 
Figures

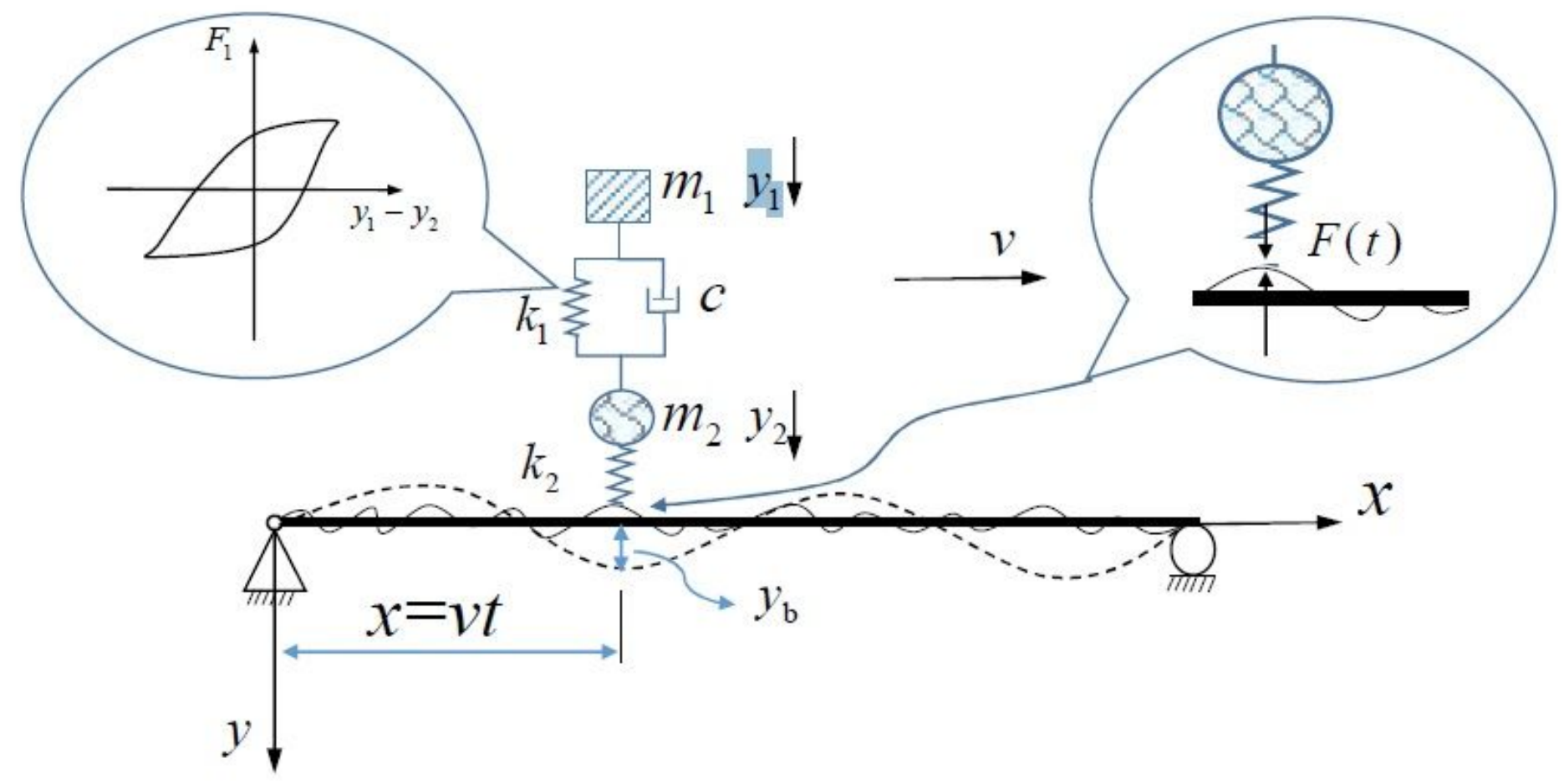

Figure 1

Mechanical model of a coupled vehicle-bridge system

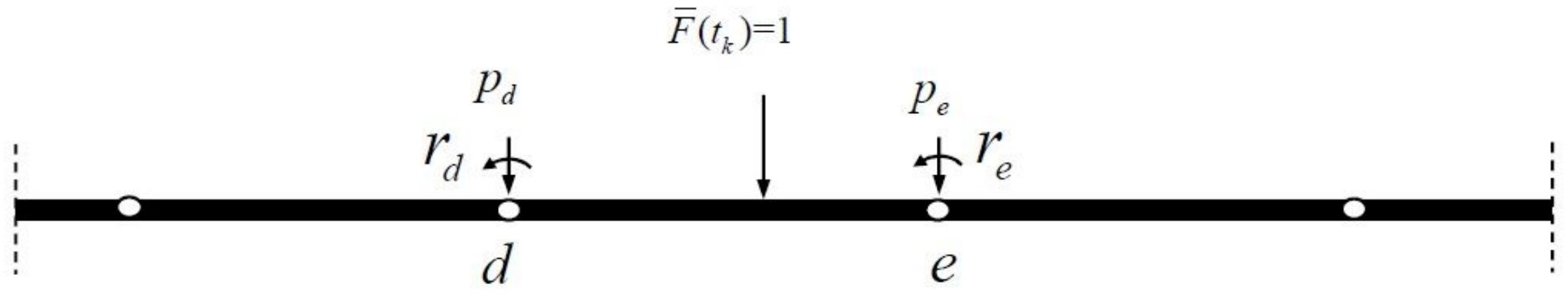

Figure 2

Schematic diagram of the wheel-bridge contact position in FEM model 
Obtain Eq. (20) based on Eq.

(11) at all time instants.

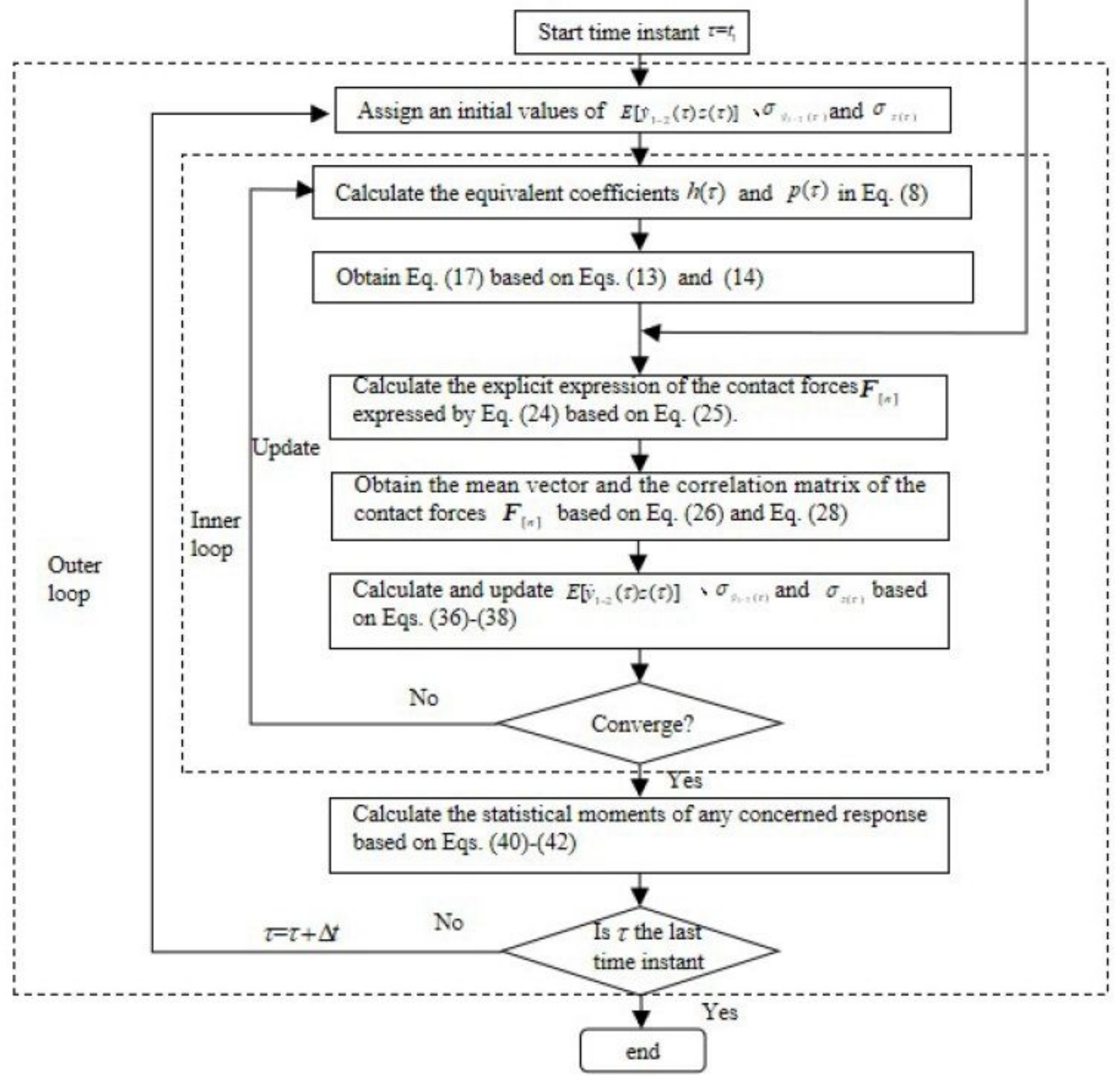

\section{Figure 3}

The flowchart of the solution procedure based on the proposed method 


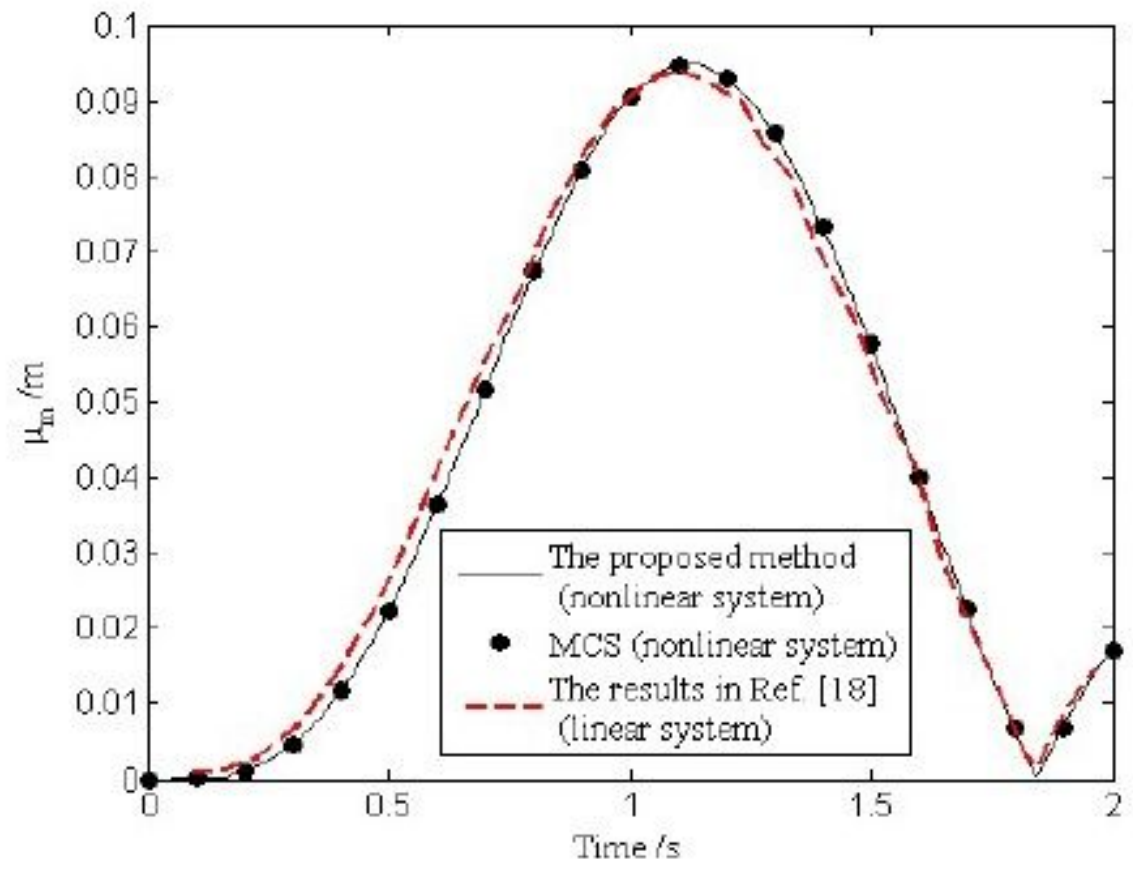

Figure 4

The mean time history for the mid-span displacement $(a=0.5)$

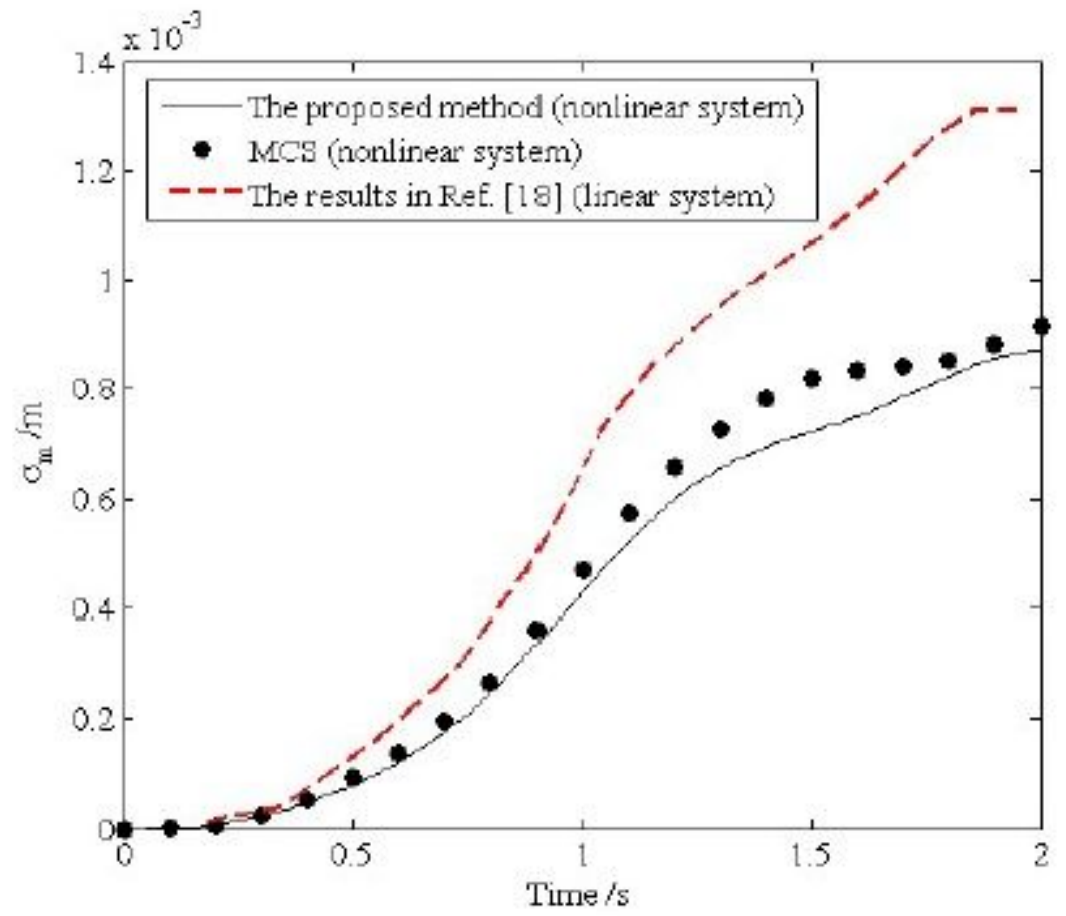

Figure 5

The standard time history for the mid-span displacement $(a=0.5)$ 


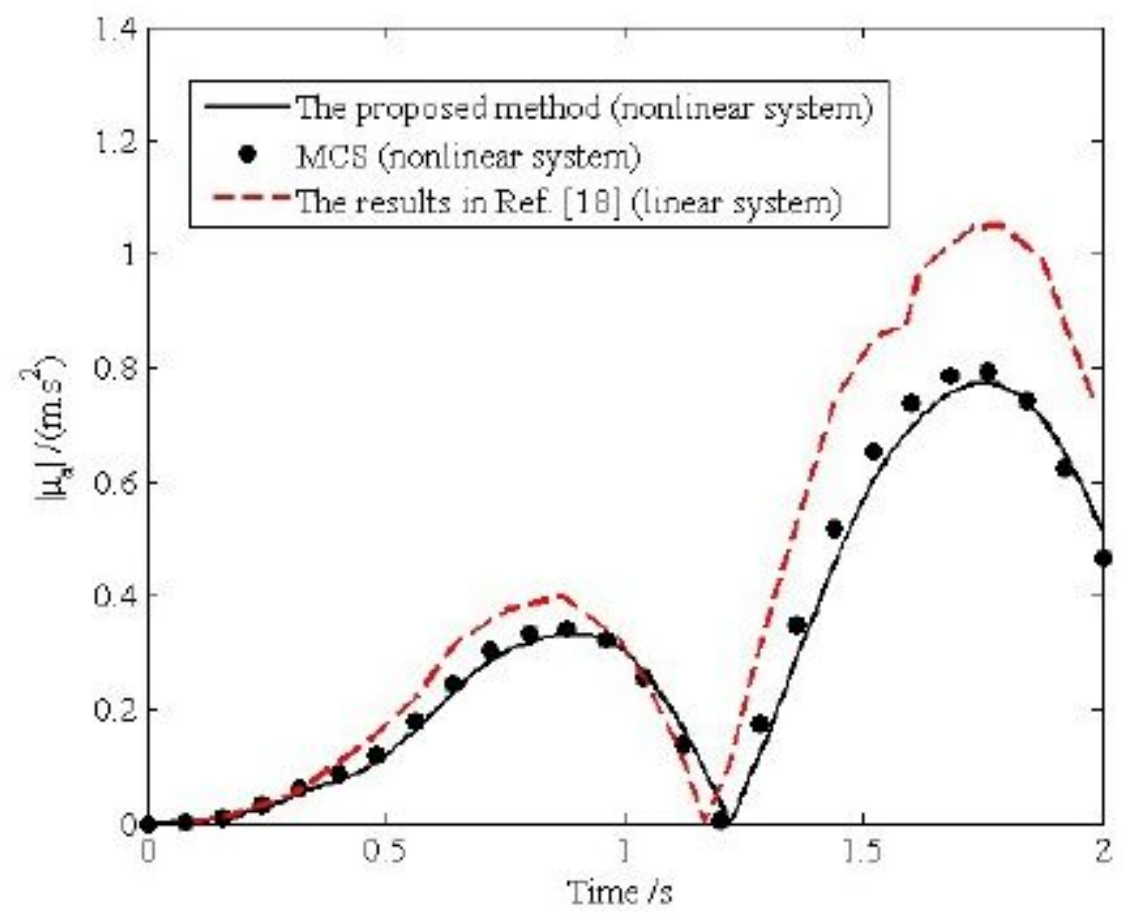

Figure 6

The mean value time history of vertical acceleration a of vehicle body $1 \mathrm{~m}(\mathrm{a}=0.5)$

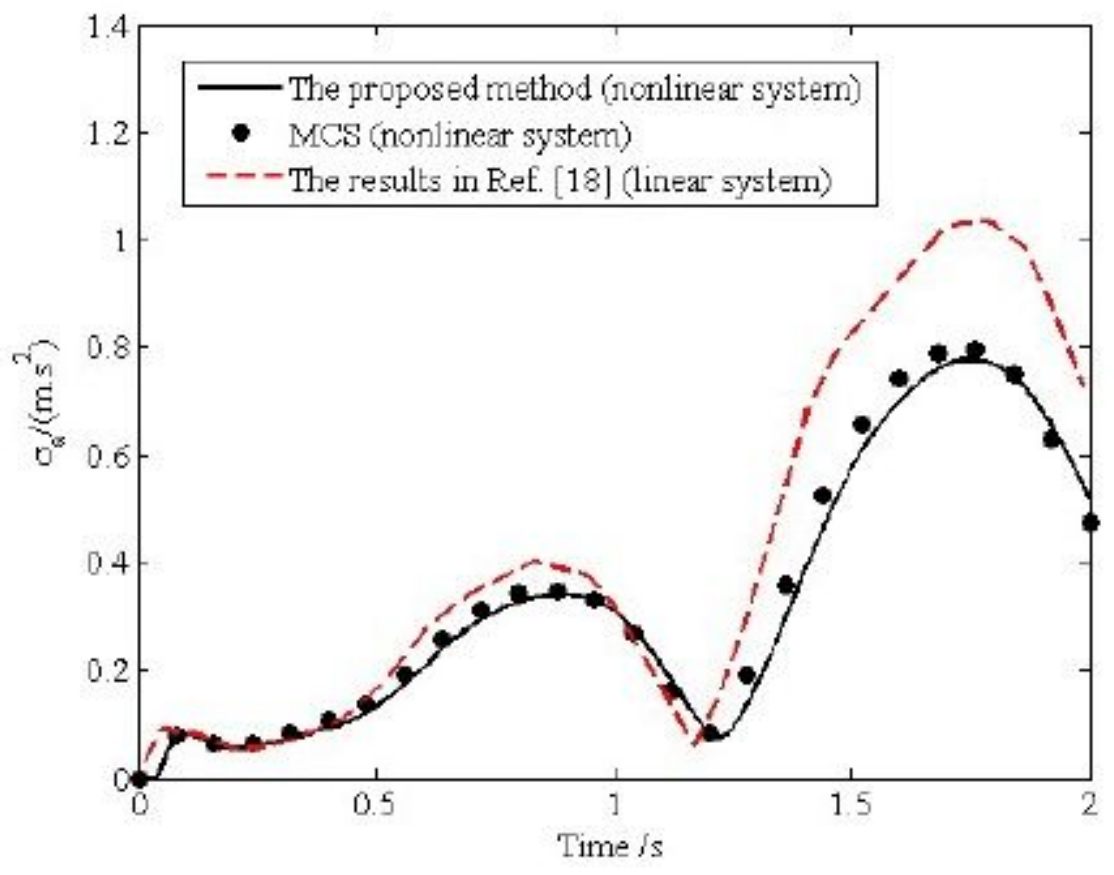

Figure 7

The standard time history of vertical acceleration a of vehicle body $1 \mathrm{~m}(\mathrm{a}=0.5)$ 


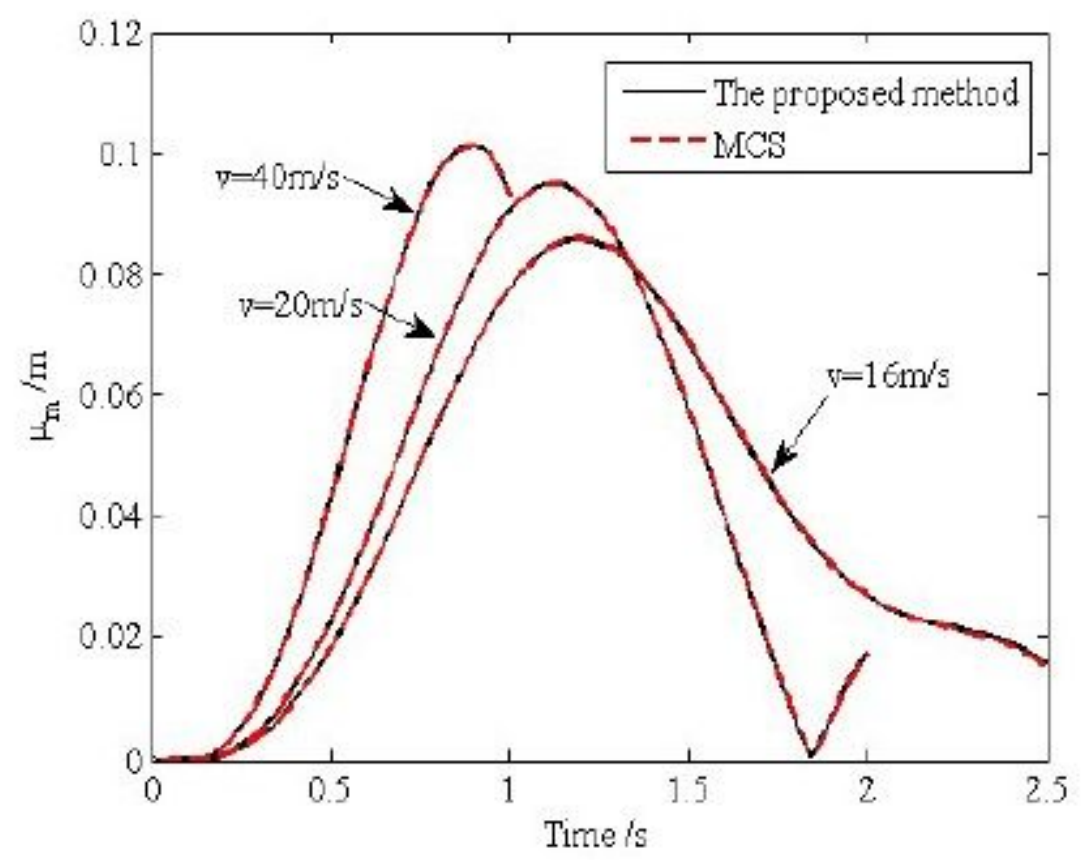

Figure 8

The mean time histories for the mid-span displacement with different velocities ( $a=0.5$ )

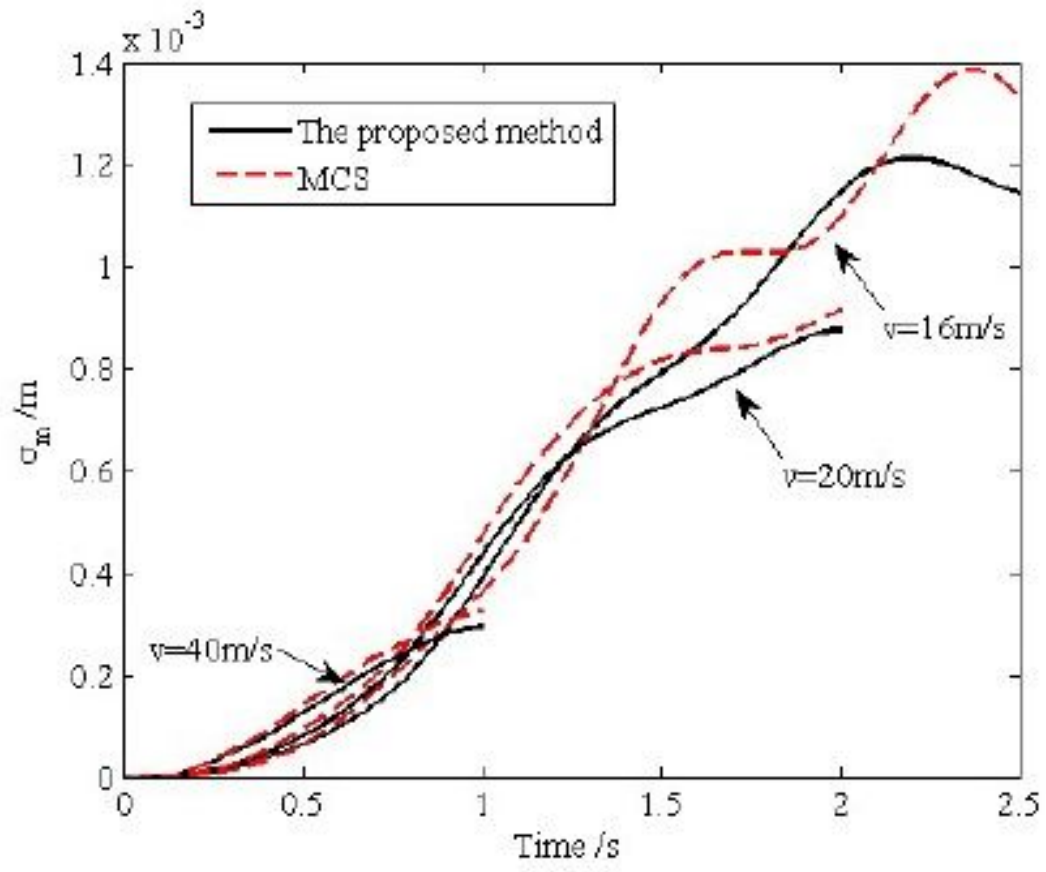

Figure 9

The standard time histories for the mid-span displacement with different velocities $(a=0.5)$ 


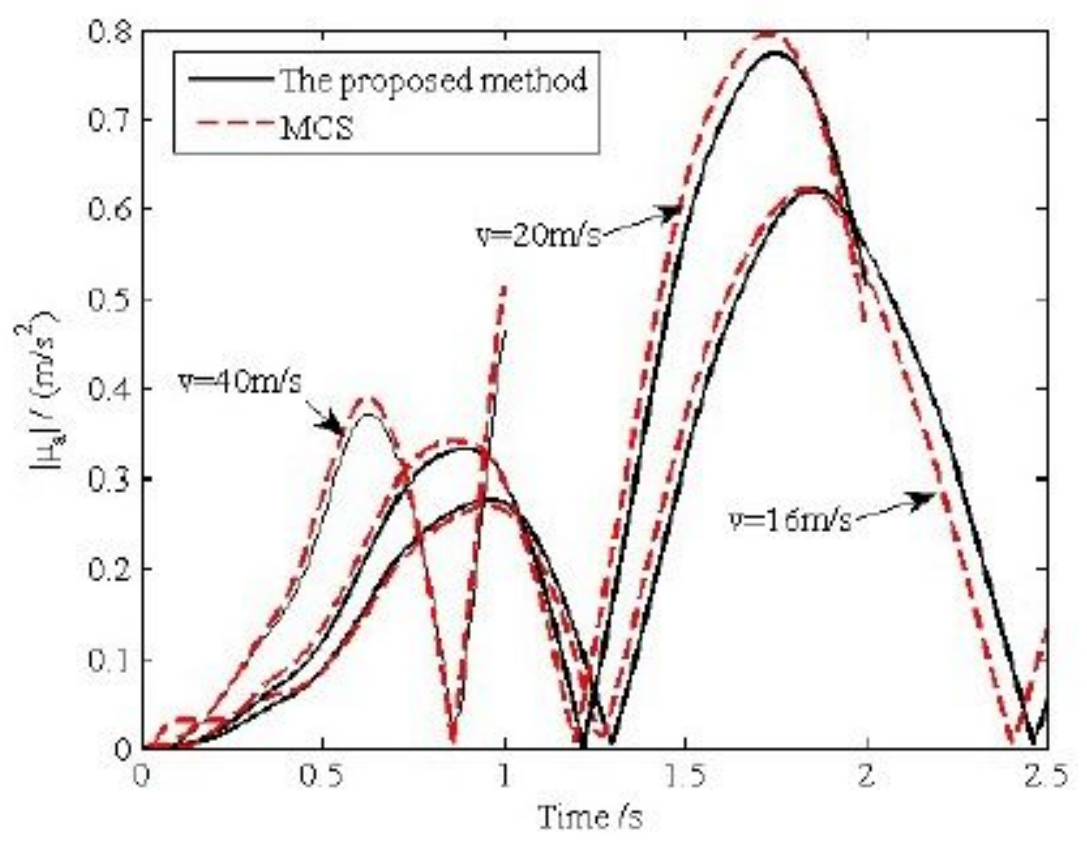

Figure 10

The mean value time histories of vertical acceleration a of vehicle body $1 \mathrm{~m}$ with different velocities (a $=0.5$ )

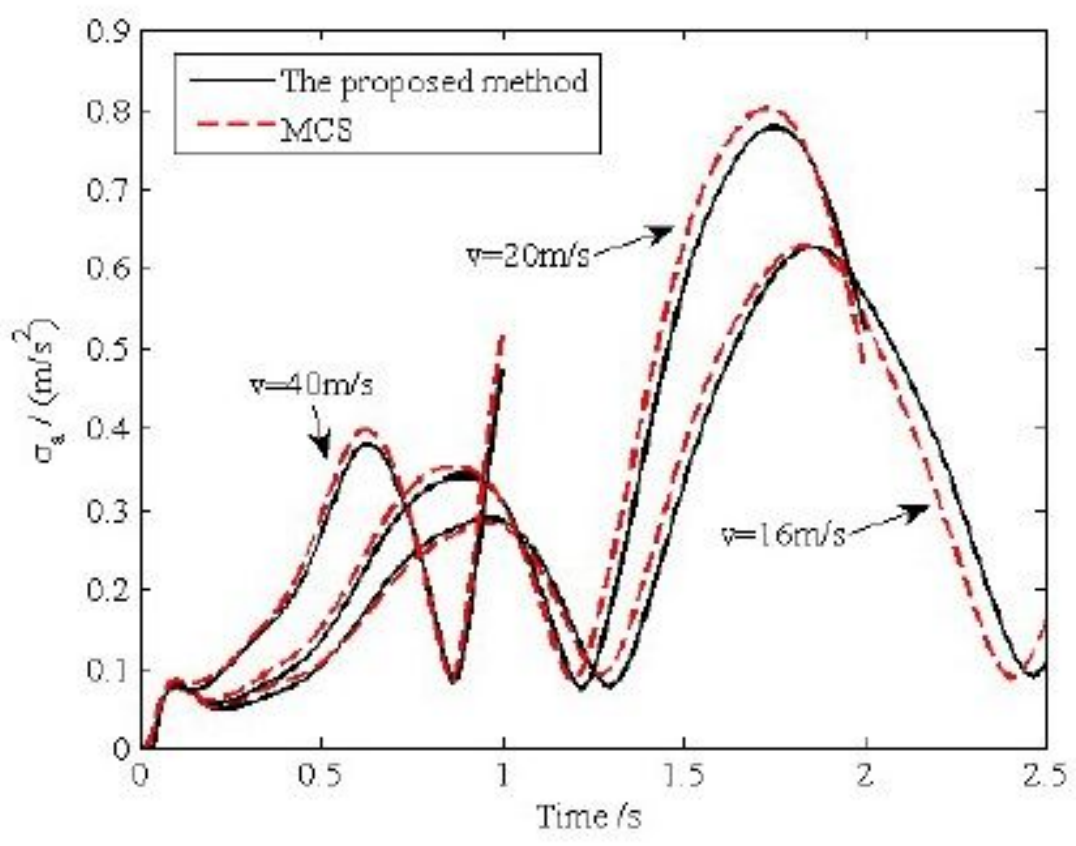

Figure 11

The standard time histories of vertical acceleration a of vehicle body $1 \mathrm{~m}$ with different velocities $(\mathrm{a}=0.5)$ 


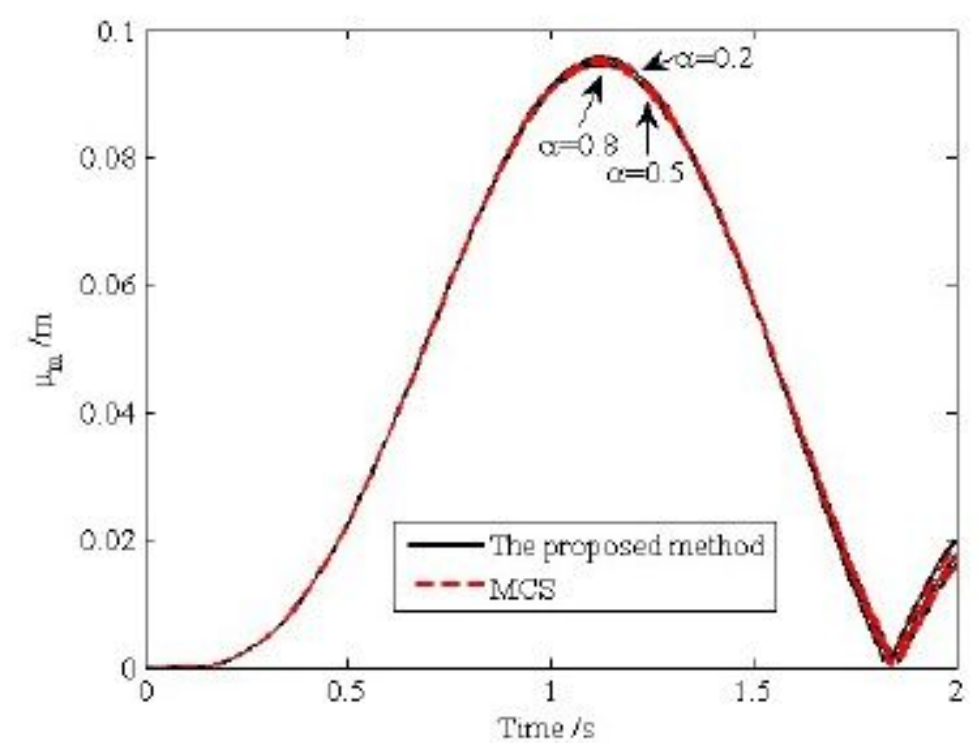

Figure 12

The mean time histories for the mid-span displacement with $a=0.8,0.5$ and 0.2

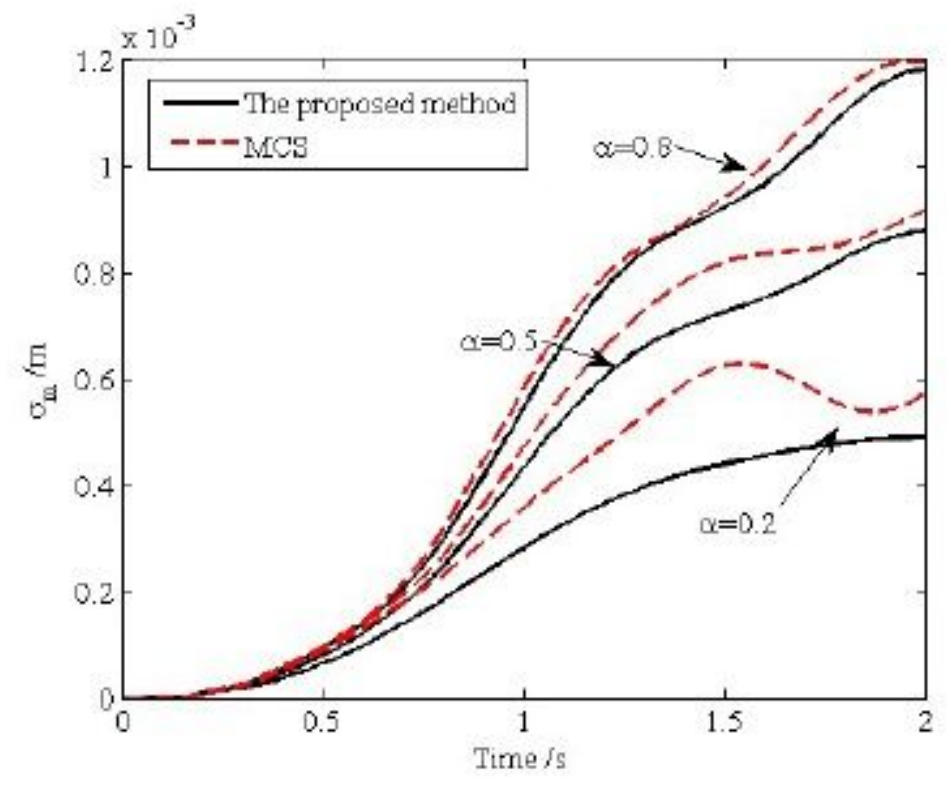

Figure 13

The standard time histories for the mid-span displacement with $a=0.8,0.5$ and 0.2 


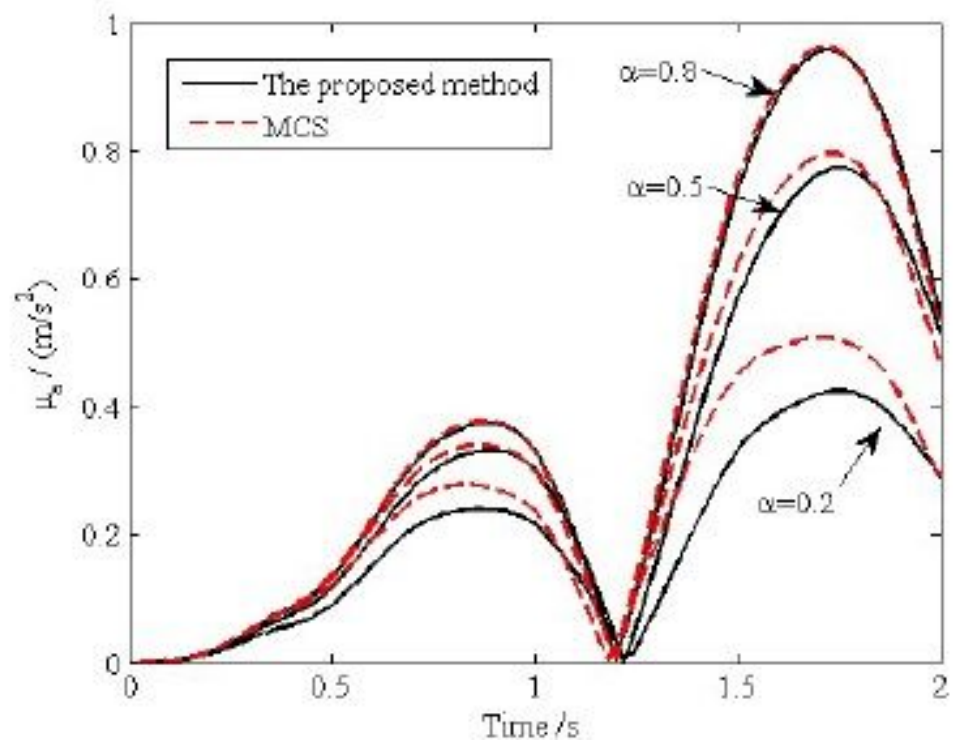

Figure 14

The mean value time histories of vertical acceleration a of vehicle body $1 \mathrm{~m}$ with $\mathrm{a}=0.8,0.5$ and 0.2

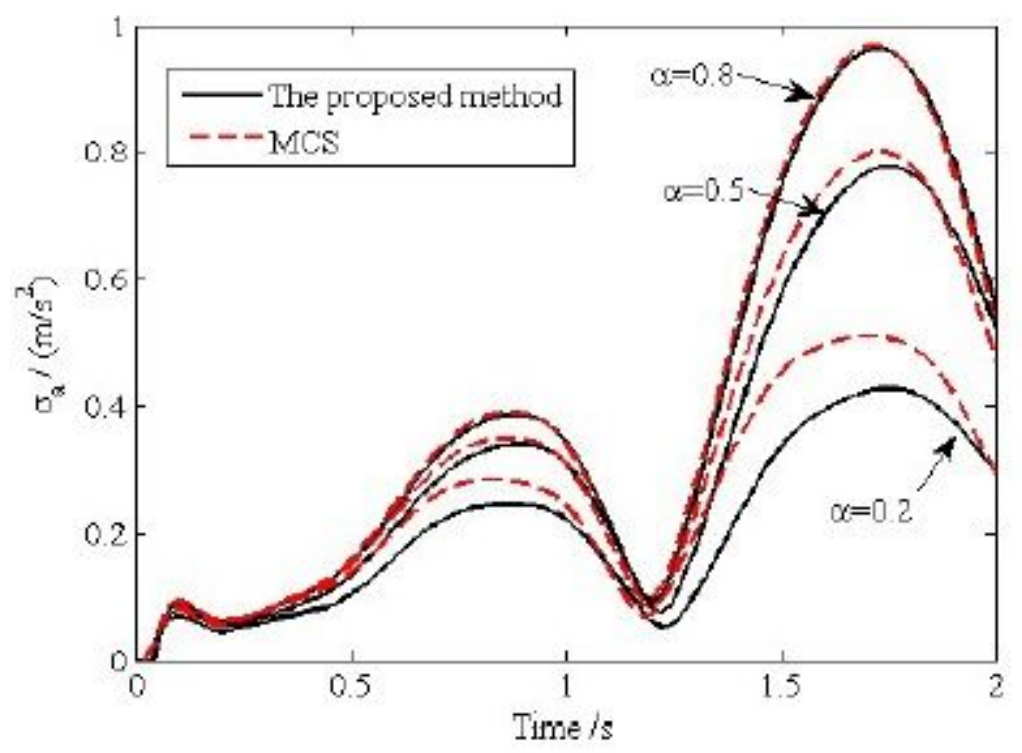

Figure 15

The standard time histories of vertical acceleration a of vehicle body $1 \mathrm{~m}$ with $\mathrm{a}=0.8,0.5$ and 0.2 